Département de sciences économiques

9815

Asymptotic Approximations in the Near-Integrated Model with a Non-Zero Initial Condition

\title{
PERRON, Pierre
}

VODOUNOU, Cosme 


\section{Département de sciences économiques}

Université de Montréal

Faculté des arts et des sciences

C.P. 6128, succursale Centre-Ville

Montréal (Québec) H3C 3J7

Canada

http://www.sceco.umontreal.ca

SCECO-information@UMontreal.CA

Téléphone : (514) 343-6539

Télécopieur : (514) 343-7221

Ce cahier a également été publié par le Centre interuniversitaire de recherche en économie quantitative (CIREQ) sous le numéro 3297.

This working paper was also published by the Center for Interuniversity Research in Quantitative Economics (CIREQ), under number 3297.

ISSN 0709-9231 


\title{
CAHIER 9815
}

\section{ASYMPTOTIC APPROXIMATIONS IN THE NEAR-INTEGRATED MODEL WITH A NON-ZERO INITIAL CONDITION}

\author{
Pierre PERRON ${ }^{1}$ and Cosme VODOUNOU ${ }^{2}$
}

1 Centre de recherche et développement en économique (C.R.D.E.) and Département de sciences économiques, Université de Montréal, and Department of Economics, Boston University

2 Institut National de la Statistique et de l'Analyse Économique, Bénin

December 1997

This paper is drawn from chapter 1 of Vodounou's Ph.D. Dissertation at the Université de Montréal. Perron acknowledges financial support from the Social Sciences and Humanities Research Council (SSHRC) of Canada, the Natural Sciences and Engineering Research Council (NSERC) of Canada, and the Fonds pour la Formation de chercheurs et l'aide à la recherche (FCAR) of Québec. The authors also thank Jon Knight for useful conversations and for providing them with the draft notes referred to as Knight and Satchell (1990). 


\section{RÉSUMÉ}

Ce papier considère plusieurs approximations asymptotiques dans le modèle autorégressif de premier ordre presque intégré avec une condition initiale non nulle. En premier, nous élargissons le travail de Knight et Satchell (1993) qui ont considéré le cas d'une marche aléatoire avec une condition initiale nulle. Ensuite, nous dérivons l'expansion de la fonction génératrice de moments conjointe qui est pertinente dans ce cadre théorique plus général. Nous considérons aussi, comme approximation alternative, l'expansion stochastique de Phillips (1987c) et l'approximation en temps continu de Perron (1991). Nous assurons comment ces méthodes alternatives donnent ou non une approximation adéquate pour la distribution en échantillon fini de l'estimateur de moindres carrés dans un modèle $A R(1)$. Les résultats montrent que, quand la condition initiale est non nulle, l'approximation en temps continu de Perron (1991) fonctionne très bien, alors que les autres offrent seulement des améliorations lorsque la condition initiale est nulle.

Mots clés: expansion d'Edgeworth, asymptotiques en temps continu, expansion stochastique, fonction de répartition, modèle autorégressif

\section{ABSTRACT}

This paper considers various asymptotic approximations in the near-integrated firstorder autoregressive model with a non-zero initial condition. We first extend the work of Knight and Satchell (1993), who considered the random walk case with a zero initial condition, to derive the expansion of the relevant joint moment generating function in this more general framework. We also consider, as alternative approximations, the stochastic expansion of Phillips (1987c) and the continuous time approximation of Perron (1991). We assess how these alternative methods provide or not an adequate approximation to the finite-sample distribution of the least-squares estimator in a first-order autoregressive model. The results show that, when the initial condition is non-zero, Perron's (1991) continuous time approximation performs very well while the others only offer improvements when the initial condition is zero.

Key words : Edgeworth expansion, continuous-time asymptotics, stochastic expansion, distribution function, autoregressive model 


\section{Introduction}

It is often the case that a nuisance parameter has a substantial effect on the distribution of some statistic in finite samples but that asymptotically this effect disappears. For that reason, several refinements of the usual asymptotic framework have been suggested which retain the effect of such nuisance parameters. A standard example is the Edgeworth expansion which has been applied to several cases. In this paper, we consider the standard first-order autoregressive model where the initial condition is one such nuisance parameter that substantially affects the finite sample distribution of the least-squares estimator but whose effect dissipates asymptotically.

The autoregressive model of order one has attracted considerable attention in both the statistics and econometrics literature, especially concerning the study of various asymptotic refinements. It is simple enough to yield tractable solutions, yet complex enough to generate interesting problems. It is somewhat of a base case by which we can learn useful features about the properties of alternative asymptotic refinements that can be helpful in guiding our efforts in more complex and empirically relevant models.

Since the pioneering works of Mann et Wald (1943) and Rubin (1950) establishing the root-T asymptotic normality of the least-squares estimator in the stationary case much of the research has focused on the case where the autoregressive coefficient, say $\alpha$, is equal to one; see White (1958), Fuller (1976), Dickey et Fuller (1979), Evans et Savin (1981), Phillips (1987a), and Phillips et Perron (1988) among others. A feature of interest in this case is that the rate of convergence is $T$ and that the limit distribution can be expressed in terms of a functional of a Weiner process. Also, White (1958) has shown in the explosive case that the rate of convergence is even faster (and depends on $\alpha$ ), and that the limit distribution is non-standard and depends on the value of the initial condition. When the latter is zero the limit is that of a Cauchy random variable.

Two features emerge from these asymptotic results which contrast with the finite sample distribution of the estimator: the discontinuity (of both the rate of convergence and the limit) at the boundary $\alpha=1$ and the invariance of the distribution with respect to the initial condition (unless $\alpha$ is greater than one). Since the finite sample distribution is continuous with respect to $\alpha$ and is influenced by the initial condition, this suggests that these limiting distributions should be poor approximations to the finite sample distribution when $\alpha$ is not equal but near to one. This problem has effectively been demonstrated by Evans and Savin (1981). 
To alleviate the first problem concerning the discontinuity at one, the research has concentrated on the so-called near-integrated model where the autoregressive coefficient is modeled as being local to unity, for example using the specification $\alpha=\exp (c / T)$ where $T$ is the sample size and $c$ some non-centrality parameter. When $c=0$, we recover the unit root case; when $c<0$, the process is said to be locally stationary and when $c>0$, it is locally explosive. The resulting limiting distribution of the least-squares estimator is continuous with respect to the non-centrality parameter $c$ and can be expressed as a functional of an Ornstein-Uhlenbeck process. Also, it provides a very good approximation to the finite sample distribution when the initial condition is zero. See Bobkoski (1983), Cavanagh (1986), Chan et Wei (1987), Phillips (1987b) and Perron (1989). The limiting distribution remains, however, invariant with respect to the initial condition and, accordingly, the adequacy of the approximation deteriorates when this initial value is non-zero.

A few asymptotic approximations have been proposed to capture the effect of this initial condition while retaining the continuity at $\alpha=1$. One is the stochastic expansion of Phillips (1987c) and the other is the continuous time approximation of Perron (1991).

This paper contributes to this literature by first considering the Edgeworth expansion in this near-integrated context. Phillips (1977) showed that in the stationary case the Edgeworth expansion is not satisfactory when the autoregressive coefficient is close to one. Satchell (1984) considered the Edgeworth expansion to order $O\left(T^{-1 / 2}\right)$ for the case of a fixed initial condition in the stationary and explosive cases and Vodounou (1997b) extended his results to order $O\left(T^{-1}\right)$ for the stationary case (the expansion to order $O\left(T^{-1}\right)$ is necessary for the initial condition to affect the distribution). Also, Knight and Satchell (1993) have shown that the Edgeworth expansion performs well in the unit root case when the initial condition is zero. Our aim is to see how it performs in the general near-integrated case with a non-zero initial condition and compare its relative merits, as an approximation to the finite sample distribution, with Phillip's stochastic expansion and Perron's continuous time approximation.

The rest of this paper is structured as follows. Section 2 presents the model and the estimator as well as some finite sample distributional results. Section 3 presents the three asymptotic approximations and the relevant moment generating functions used to numerically evaluate the corresponding distribution functions. Section 4 contains a study of the adequacy of the various approximations for a selected range of cases. Section 5 offers some concluding comments and a mathematical appendix some technical derivations. 


\section{Model and Estimator}

We consider the $\mathrm{AR}(1)$ model defined as follows:

$$
\begin{gathered}
y_{t}=\alpha y_{t-1}+u_{t}, \\
u_{t} \sim N\left(0, \sigma^{2}\right), \\
\alpha=\exp (c / T), \\
y_{0} / \sigma=\kappa \quad \text { fixed, } \\
t=1, \ldots, T .
\end{gathered}
$$

Here the scaled initial value $y_{0} / \sigma$ is considered fixed at some constant $\kappa$, the errors $u_{t}$ are independent and normally distributed with mean 0 and variance $\sigma^{2}$ and $c$ is some real fixed coefficient. Here, the autoregressive coefficient $\alpha$ is said to be local to unity. When $c=0$, we have a unit root; when $c<0$, the process is locally stationary, and when $c>0$ it is locally explosive. We consider the $O L S$ estimator of $\alpha$ defined by:

$$
\widehat{\alpha}=\sum_{t=1}^{T} y_{t} y_{t-1} / \sum_{t=1}^{T} y_{t-1}^{2} .
$$

This is also the maximum likelihood estimator conditional upon $y_{0}$. The estimate $\hat{\alpha}$ being invariant to a scale transformation of the data, we can, without loss of generality, suppose that $u_{t} \sim N(0,1)$ so that $y_{0}=\kappa$. We are interested in the distribution of the statistic:

$$
T(\widehat{\alpha}-\alpha)=\frac{T^{-1}\left(\sum_{t=1}^{T} y_{t} y_{t-1}-\alpha \sum_{t=1}^{T} y_{t-1}^{2}\right)}{T^{-2} \sum_{t=1}^{T} y_{t-1}^{2}} .
$$

Using matrix notation and the vector $y=\left(y_{1}, y_{2}, \ldots ., y_{T}\right)^{\prime}$, we can write this statistic as:

$$
T(\widehat{\alpha}-\alpha)=\frac{y^{\prime} A y / T+\left(y_{1} \kappa-\kappa^{2} \alpha\right) / T}{y^{\prime} B y / T^{2}+\kappa^{2} / T^{2}}
$$

where $A$ and $B$ are $T$ by $T$ symmetric matrices (and $B$ is positive definite) defined by (see, e.g., White (1958), Evans et Savin (1981b), Satchell (1984), and Knight and Satchell (1993)):

$$
A=\left[\begin{array}{ccccc}
-\alpha & 1 / 2 & 0 & \ldots & 0 \\
1 / 2 & -\alpha & \ddots & \ddots & \vdots \\
0 & 1 / 2 & \ddots & \ddots & 0 \\
\vdots & \ddots & \ddots & -\alpha & 1 / 2 \\
0 & \cdots & 0 & 1 / 2 & 0
\end{array}\right]
$$


and

$$
B=\left[\begin{array}{cc}
I_{T-1} & \widetilde{O}^{\prime} \\
\widetilde{O} & 0
\end{array}\right]
$$

where $I_{T-1}$ is the identity matrix of dimension $T-1$ and $\widetilde{O}$ is a 1 by $(T-1)$ vector of zeros. We use the notation:

$$
\begin{aligned}
& X=y^{\prime} A y / T+\left(y_{1} \kappa-\kappa^{2} \alpha\right) / T \\
& H=y^{\prime} B y / T^{2}+\kappa^{2} / T^{2}
\end{aligned}
$$

and define the joint moment generating function of $(X, H)$ by $m_{\kappa}(u, v)=E(\exp \{u X+v H\})$. From the result of White (1958), we have:

$$
m_{\kappa}(u, v)=\exp \left\{\kappa^{2}\left(\frac{v}{T^{2}}-\alpha \frac{u}{T}-\frac{\alpha^{2}}{2}\right)+\frac{\kappa^{2}}{2}\left(\frac{u}{T}+\alpha\right)^{2} \frac{D(T-1)}{D(T)}\right\} D(T)^{-1 / 2},
$$

where $D(T)$ satisfies the second order difference equation:

$$
D(T)=p D(T-1)-q^{2} D(T-2)
$$

with initial conditions $D(1)=1$ et $D(2)=p-q^{2}$ and where $p=1+\alpha^{2}-2 v / T^{2}+2 \alpha u / T$ and $q=-(\alpha+u / T)$. Solving this system, we have:

$$
D(T)=\frac{1-s}{r-s} r^{T}+\frac{1-r}{s-r} s^{T}
$$

where $r$ and $s$ are the roots of the equation $x^{2}-p x+q^{2}=0$. Expression (7) allows to compute the joint characteristic function of the pair $(X, H)$ and, using Gurland's (1948) inversion theorem, to obtain the exact distribution of the statistic $T(\hat{\alpha}-\alpha)$.

\section{Alternative asymptotic approximations}

We now discuss three different asymptotic approximations which apply to this near-integrated context and deliver distributions that are specifically influenced by the value of the initial condition. These are: an extension of the Edgeworth approximation studied by Knight and Satchell (1993), the stochastic expansion considered by Phillips (1987c) and the continuoustime approximation analyzed by Perron (1991). 


\subsection{The Edgeworth approximation}

Here, we extend the work of Knight and Satchell (1993) who considered the case $c=0$ with a zero initial condition. We first consider the joint moment generating function of the pair $(X, H)$ defined by $(5)$ and $(6)$. The result is given in the following theorem ${ }^{1}$.

Theorem 1 Let $\left\{y_{t}\right\}$ be generated by (1) and denote by $M G F(u, v)$ the joint momentgenerating function of the pair $(X, H)$ defined by (5) and (6), then:

$$
\begin{aligned}
M G F(u, v)= & \exp \left(-r_{1} / 2\right)\left(\cos \delta-r_{1} \frac{\sin \delta}{\delta}\right)^{-1 / 2} \\
& \times\left\{1+\frac{F_{1}(u, v, c)}{4 T} \frac{\sin \delta}{\delta}+\kappa^{2} \frac{F_{2}(u, v, c)}{2 T} \frac{\sin \delta}{\delta}-c^{2} \frac{F_{3}(u, v, c)}{4 T} \cos \delta\right\}+O\left(T^{-2}\right)
\end{aligned}
$$

where

$$
\begin{aligned}
& F_{1}(u, v, c)=\frac{\left(u^{2}+2 v\right)\left(2 c^{2}+c^{3}-2 u v+2 c u^{2}+2 c u-2 v-2 c v+3 c^{2} u\right)}{\delta^{2}\left(\cos \delta-r_{1} \frac{\sin \delta}{\delta}\right)}, \\
& F_{2}(u, v, c)=\frac{u^{2}+2 v}{\cos \delta-r_{1} \frac{\sin \delta}{\delta}}, \\
& F_{3}(u, v, c)=\frac{u^{2}+2 v}{\delta^{2}\left(\cos \delta-r_{1} \frac{\sin \delta}{\delta}\right)},
\end{aligned}
$$

and $r_{1}=c+u, \delta^{2}=2 v-c u+c^{2}$.

The proof is presented in the appendix. In what follows we shall denote by $M G F_{K S}(u, v)$ the approximation to the joint moment-generating function of the pair $(X, H)$ obtained by retaining terms that are of higher order than $O\left(T^{-2}\right)$. That is, we have:

$$
\begin{aligned}
M G F_{K S}(u, v)= & \exp \left(-r_{1} / 2\right)\left(\cos \delta-r_{1} \frac{\sin \delta}{\delta}\right)^{-1 / 2} \\
& \times\left\{1+\frac{F_{1}(u, v, c)}{4 T} \frac{\sin \delta}{\delta}+\kappa^{2} \frac{F_{2}(u, v, c)}{2 T} \frac{\sin \delta}{\delta}-c^{2} \frac{F_{3}(u, v, c)}{4 T} \cos \delta\right\} .
\end{aligned}
$$

The use of Gurland's (1948) inversion theorem leads to the following approximation for the distribution function $\operatorname{Pr}(T(\widehat{\alpha}-\alpha)<z)$ :

$$
F_{K S}(z)=\frac{1}{2}-\frac{1}{\pi} \int_{0}^{\infty} I M\left(\frac{M G F_{K S}(i u,-i u z)}{u}\right) d u
$$

\footnotetext{
${ }^{1}$ The expansion presented below and it's proof were contained in most parts in some draft notes, Knight and Satchell (1990). We, nevertheless, present the full proof in the appendix since that draft contained some technical errors (hence, a different expression) and was never published.
} 
where $I M($.$) denotes the imaginary part of the argument { }^{2}$. We close by noting that the specification $\alpha=\exp (c / T)$ instead of $\alpha=1+c / T$ is not innocuous. Use of the latter would have given a slightly different expression for the joint moment-generating function.

\subsection{The stochastic expansion}

We consider now the stochastic expansion derived by Phillips (1987c) and also studied by Perron (1996). From the latter, we have:

$$
T(\widehat{\alpha}-\alpha) \stackrel{d}{=} Z(c, \gamma) / K(c, \gamma)+O_{p}\left(T^{-1}\right)
$$

where

$$
\begin{aligned}
Z(c, \gamma) & =\int_{0}^{1} J_{c}(r) d W(r)+\gamma \int_{0}^{1} \exp (c r) d W(r)-\eta / \sqrt{2 T} \\
K(c, \gamma) & =\int_{0}^{1} J_{c}^{2}(r) d r+2 \gamma \int_{0}^{1} \exp (c r) J_{c}(r) d r
\end{aligned}
$$

Here, $\eta$ is a $N(0,1)$ random variable independent of the Weiner process $W(r), J_{c}(r)$ is the Ornstein-Uhlenbeck process defined by $J_{c}(r)=\int_{0}^{r} \exp (c(r-s)) d W(s)$ and $\gamma=\kappa / T^{1 / 2}$. Here we approximate the distribution of $T(\hat{\alpha}-\alpha)$ by the distribution of the random variable $Z(c, \gamma) / K(c, \gamma)$. The joint moment generating function of the pair $(Z(c, \gamma), K(c, \gamma))$ was derived by Perron (1996) in a more general context and it is shown in the appendix that it can be represented as:

$$
\begin{aligned}
M G F_{P C B}(u, v)= & \exp \left(-r_{1} / 2\right)\left(\cos \delta-r_{1} \frac{\sin \delta}{\delta}\right)^{-1 / 2} \exp \left\{\frac{u^{2}}{4 T}-\frac{\exp (2 c)-1}{2 c} v \gamma^{2}\right\} \\
& \times \exp \left\{-\frac{\gamma^{2} v}{2}\left(r_{1}-\lambda\right)\left[1-\frac{\exp (-\lambda)}{\cos \delta-\frac{r_{1}}{\delta} \sin \delta}\right]\right\}
\end{aligned}
$$

where $\delta$ and $r_{1}$ are defined in Theorem 1 and $\lambda^{2}=-\delta^{2}$. The corresponding approximation to the distribution function $T(\hat{\alpha}-\alpha)$ is obtained using the inversion formula (10) with $M G F_{P C B}(u, v)$ instead of $M G F_{K S}(u, v)$ in which case the distribution function is denoted $F_{P C B}(z)$.

\footnotetext{
${ }^{2}$ As in Knight and Satchell (1993), we can write the distribution function as $F_{K S}(z)=1 / 2$ $-(1 / \pi)\left[F U N C D 0(z)+(1 / 4 T) F U N C D 1(z)+\left(c^{2} / 4 T\right) F U N C D 2(z)-\left(\kappa^{2} / 2 T\right) F U N C D 3(z)\right]$. In practice, the four functions, FUNCDi(z) are numerically integrated separately and the final value of the distribution function obtained using this relation. The values of these functions for a grid of $z$ 's are presented in Vodounou (1997a). They are not reproduced here to conserve space.
} 
The expansion (11) is not, strictly, a stochastic expansion to order $O_{p}\left(T^{-1}\right)$ since it retains some terms that are $O_{p}\left(T^{-1}\right)$. The expansion that keeps only terms that are of order $O_{p}\left(T^{-1 / 2}\right)$ is:

$$
\frac{Z(c, \gamma)}{K(c, \gamma)}=X_{0}+X_{1} T^{-1 / 2}+O_{p}\left(T^{-1}\right)
$$

where

$$
\begin{aligned}
& X_{0}=H_{0} / K_{0} \\
& X_{1}=\frac{1}{K_{0}}\left(H_{1}-K_{1} \frac{H_{0}}{K_{0}}\right)
\end{aligned}
$$

with $H_{0}=\int_{0}^{1} J_{c}(r) d W(r), H_{1}=\kappa \int_{0}^{1} \exp (c r) d W(r)-\eta / \sqrt{2}, K_{0}=\int_{0}^{1} J_{c}(r)^{2} d r$, and $K_{1}=2 \kappa \int_{0}^{1} \exp (c r) J_{c}(r) d r$. However, obtaining the moment-generating function of the variable $X_{0}+X_{1} T^{-1 / 2}$ is more complex and we continue to work with the random variable $Z(c, \gamma) / K(c, \gamma)$ as the approximation. We will see later that excluding all terms that are $O_{p}\left(T^{-1}\right)$ would deteriorate the approximation.

\subsection{Continuous time approximation}

Consider a continuous time process $y_{t}$ defined by the following stochastic differential equation:

$$
d y_{t}=\theta y_{t} d t+\sigma_{1} d W(t) \quad y_{0}=\kappa \sigma_{1} \quad t \in[0, N]
$$

where $\theta$ and $\sigma$ are unknown parameters, $b$ is a fixed constant and $N$ is the span of the data. The stochastic process $\left\{y_{t}\right\}$ has the following discrete time, representation:

$$
y_{t h}=\alpha_{h} y_{(t-1) h}+u_{t h} \quad y_{0}=\kappa \sigma_{1} \quad t=1, \ldots, T
$$

where $u_{t h} \sim N\left(0, \sigma_{1}^{2}(\exp (2 \theta h)-1) / 2 \theta\right)$ and $h$ is the sampling interval such that $T h=$ $N$ (see, e.g., Bergstrom (1984), Phillips (1987a), and Perron (1991)) The autoregressive

coefficient is $\alpha_{h}=\exp (\theta h)$. Hence, (17) is equivalent (up to a scale transformation) to model (1) with $c=\theta N$.

Perron (1991) considered the distribution of the least-squares estimators of $\hat{\theta}$ and $\hat{\alpha}_{h}$ defined, respectively, by $\hat{\theta}=\int_{0}^{N} y_{t} d y_{t} / \int_{0}^{N} y_{t}^{2} d t$ and $\hat{\alpha}_{h}=\sum_{t=1}^{T} y_{t h} y_{(t-1) h} / \sum_{t=1}^{T} y_{(t-1) h}^{2}$ when $y_{t}$ and $y_{t h}$ are generated by (16) et (17). He obtained the results:

$$
\begin{gathered}
N(\hat{\theta}-\theta) \stackrel{d}{=} A(\gamma, c) / B(\gamma, c), \\
T\left(\hat{\alpha}_{h}-\alpha_{h}\right) \Rightarrow A(\gamma, c) / B(\gamma, c),
\end{gathered}
$$


the convergence result being obtained with $N$ fixed and $h$ converging to 0 . The variables are defined by:

$$
\begin{aligned}
& A(\gamma, c)=\int_{0}^{1} J_{c}(r) d W(r)+\gamma \int_{0}^{1} \exp (c r) d W(r) \\
& B(\gamma, c)=\int_{0}^{1} J_{c}^{2}(r) d r+2 \gamma \int_{0}^{1} \exp (c r) J_{c}(r) d r+\gamma^{2}(\exp (2 c)-1) / 2 c
\end{aligned}
$$

with $\gamma=\kappa / T^{1 / 2}$ and $c=\theta N$. Here, we consider approximating the distribution of $T(\hat{\alpha}-\alpha)$ by the distribution of the random variable $A(\gamma, c) / B(\gamma, c)$ which we denote by $F_{P P}(z)$. From Perron (1991), we have

$$
F_{P P}(z)=\frac{1}{2}-\frac{1}{\pi} \int_{0}^{\infty} I M\left(\frac{M G F_{P P}(i u,-i u z)}{u}\right) d u
$$

where $M G F_{P P}(u, v)$ is the joint moment generating function of the pair $(A(\gamma, c), B(\gamma, c))$ given by:

$$
M G F_{P P}(u, v)=\exp \left(-r_{1} / 2\right)\left(\cos \delta-r_{1} \frac{\sin \delta}{\delta}\right)^{-1 / 2} \exp \left\{\frac{\gamma^{2}\left(u^{2}+v\right)}{2\left(\cos \delta-r_{1} \sin \delta / \delta\right)} \frac{\sin \delta}{\delta}\right\}
$$

where $\delta^{2}=2 v-2 c u-c^{2}$ and $r_{1}=c+u$.

\section{Adequacy of the approximations}

In this section, we discuss to what extent the different approximations described are good approximations to the exact distribution of $T(\hat{\alpha}-\alpha)$ especially when the initial condition is non-zero. To that extent, we need to numerically evaluate the distribution functions $F_{K S}(z)$, $F_{P C B}(z)$ and $F_{P P}(z)$. We start with some technical descriptions on how these are performed.

\subsection{Numerical evaluation of the distribution functions}

The evaluation of the approximate distribution functions at some point $z$ requires the numerical integration of the relevant functions. An important fact to note is that this involves two special problems. The first is related to the presence of the square root of the complex

number $\left(\cos \delta-r_{1} \frac{\sin \delta}{\delta}\right)^{-1}$. Using the principal root does not ensure the continuity of the integrand. One must therefore carefully assess which roots to select along the integration path to ensure continuity. The solution to this problem is explained in detailed in Perron (1989).

The second difficulty is how to ensure a given desired precision given that the integrand may show wide fluctuations and multiple crossings at zero. Here, we follow the suggestions 
of Knight and Satchell (1993). If the integrand shows large variations near zero but reduces rapidly when evaluated at a higher number, the integral is computed over a range $[\epsilon, \bar{V}]$, where $\epsilon$ is some small number that we set at $\epsilon=1.0 \mathrm{E}-7$ and the upper bound is such that the absolute value of the integrand evaluated at this upper bound is less than $\epsilon$ (see also Perron $(1989,1991)$ and Hisamatsu and Maekawa (1994)).

However, when the integrand shows oscillatory behavior that dissipates slowly, Knight and Satchell (1993) suggest to integrate between the points at which the integrand crosses the zero axis. Here, the upper bound of integration depends on the values of the successive integrations. The method adopted is to stop whenever an integral between two points at which the integrand crosses zero is less than $1.0 \mathrm{E}-5$.

Given the particular cases, the strategies discussed above are used to compute the distribution functions $F_{K S}(z), F_{P C B}(z)$ and $F_{P P}(z)$ for various values of $c, \kappa$ and $T$. In all cases, the distribution functions are evaluated at 50 equidistant points $z$ over the range $[-14,5]$. We exclude the point $z=-c$ since the integral is ill-defined in this case; we consider instead the evaluation at some close neighbor.

\subsection{The effects of the initial condition on the distributions.}

In this section, we consider the effect of variations in the initial condition on the finite sample distribution and the three approximations considered. To obtain the finite sample distribution, we used 30,000 replications of the statistic (3) when the model is generated by (1). We consider three values of the non-centrality parameter $c$, namely $c=-5,0$ and 2 (covering the stationary, unit root and explosive cases). For the initial condition, we consider the values $\gamma\left(=y_{0} / T^{1 / 2}\right)=0, .5,1.0$ and 2.0 .

The results are presented in Figures 1.a to 1.d for the case $T=25$. Consider first, Figure 1.a which presents the results for the exact distribution. We see that as the initial condition increases the exact distribution gets more concentrated around zero and the tails of the distributions, accordingly, get shorter. This concentration around zero is more pronounced as $c$ increases.

Consider now the effects on the asymptotic approximations. An increase in the initial condition has a similar effect on the continuous-time and Edgeworth approximations but the effect is clearly different on the approximation given by the stochastic expansion. The variations in the continuous-time approximation mirrors closely those of the exact distribution. On the other hand, when $\gamma \geq 1$, the approximation provided by the Edgeworth expansion

deteriorates in the right tail and take values greater than one so that the approximation no 
longer provides a proper distribution function. The variations, induced by changes in $\gamma$, on the approximation provided by the stochastic expansion are very much different from those of the exact distribution. For a fixed $c$, we note an increase in the left tail which becomes considerable when $\gamma$ increases. The right tail decreases as $\gamma$ increases but in a way different from the finite sample distribution, especially when $c=2$.

The effect of a change in the non-centrality parameter $c$ on the exact and approximate distributions are similar to those induced by changes in the initial condition. For a given $\gamma$, these distribution (with the exception of the stochastic expansion) becomes more concentrated around zero as $c$ increases and this concentration becomes more important as $\gamma$ increases.

\subsection{Adequacy of the approximations}

The quality of the approximations depend on the values of the parameters $c$ and $\gamma$. To analyze this issue we compare the various approximations to the exact distribution for $T=10,25,50$ and 100 and for the same values of $c$ and $\gamma$ considered above. The results are presented in Tables 1 to 4 and Figure 2.a to 2.d. We consider in turn the case where $\gamma=0$ and the case where $\gamma$ is non-zero.

\subsubsection{Case with $\gamma=0$}

In the case where the initial condition is zero the continuous-time approximation is equivalent to the standard asymptotic distribution without any correction factor. This is not the case for the approximations based on the Edgeworth and the stochastic expansions which provide finite sample correction factors. The adequacy of the usual asymptotic distribution in the case of a zero initial condition was studied in detail in Perron (1989). He showed that it is not particularly adequate when $T=10$ or 25 and $c=-5$ but that the adequacy increases when $c$ increases. In particular, when $T=25$ and $c=-1$, it is satisfactory in the right tail and excellent overall when $c=5$ and $T=10$. This study also showed that the usual limiting distribution is satisfactory even with $c<0$ when $T$ reaches 50 .

As seen in Figures 2, the Edgeworth expansion, unlike the stochastic expansion, provides an improvement over the standard asymptotic (or continuous-time) distribution when $T=$ 10 or 25 , especially in the locally stationary $(c<0)$ or integrated $(c=0)$ cases. When $T=10$ and $c=-5$, the stochastic expansion is a bad approximation (Table 4) while the continuous-time and Edgeworth approximations are satisfactory in the right tail. The Edgeworth expansion is the best approximation in the left tail. When $T=25$, the Edgeworth 
approximation is excellent, the continuous time approximation is good in the right tail but less so in the left and the stochastic expansion is less good overall. As $c$ increases while remaining negative (e.g., $c=-2$ and -1 ), the continuous-time and stochastic expansion approximations remains satisfactory in the right tail but the Edgeworth expansion is best overall, especially if $T=25$ (Figures $2(\mathrm{a}, \mathrm{b}, \mathrm{c})$ ). Similar conclusions apply with a unit root $(c=0)$. When the model is locally explosive $(c>0)$, the three approximations share the common feature of being excellent for $T=25$ and $c=2$ (Figure 2.d). However, for $T=10$, the continuous-time and stochastic expansion approximations are not so good in the extreme tails. But these shortcomings disappears when $c$ increases.

\subsubsection{Case with $\gamma \neq 0$}

When the initial condition is non-zero, the continuous-time approximation is, in general, the better one. Indeed, when $T \geq 25, \gamma$ fixed and $c<0$, the continuous-time approximation is satisfactory, especially in the right tail (Figures $2(a, b)$ ). Furthermore, the quality of this approximation increases when $c$ increases. For example, when $c=2$ and $\gamma=2$ (Figure 2.d), it is excellent for $T \geq 10$. Similar conclusions apply when considering a fixed $c$ and varying $\gamma$; i.e. a good approximation in the right tail and significant improvements in the left tail as $\gamma$ increases. In particular, when $T=50$, the continuous-time approximation is excellent for $c=2$ and any $\gamma$.

The Edgeworth and stochastic expansions offer a contrasting picture, especially when $\gamma \geq 1$ (for all $T$ considered). Figures 2 show that as the initial condition increases, these approximations seriously deteriorates. For moderate values (e.g., $\gamma=0.5$ ), the stochastic expansion is still not a good approximation. On the other hand, the Edgeworth expansion is satisfactory in the right tail and seems to better capture the median of the exact distribution when $T=10$ or 25 . When $T$ increases (e.g., $T=50$ ) with $c<0$, the continuous-time and Edgeworth approximations are good in the right tail but somewhat lacking in the left. The quality of the approximation increases as $c$ increases most notably for the continuous-time approximation which is excellent when $c=-1$ or $c \geq 0$.

\subsubsection{Some analytical explanations}

It is instructive to consider the behavior of the various approximations in the case of a large initial condition, i.e. a large value of $\gamma$ (which is here equivalent to a small- $\sigma$ approximation). From Perron (1991), we have that, when $\gamma$ is large, the continuous-time approximation 
implies that

$$
T(\hat{\alpha}-\alpha) \stackrel{a}{\sim} \mathcal{N}\left(0,2 c / \gamma^{2}(\exp (2 c)-1)\right)
$$

This approximation is consistent with the main features of the finite sample distribution. In particular, it implies that as $\gamma$ increases the distribution of $T(\hat{\alpha}-\alpha)$ becomes more symmetric (due to the normality) and more concentrated around zero (since the variance decreases). Also, the approximation (20) is consistent with the finite sample feature that this concentration is more rapid (as $\gamma$ increases) the large $c$ is.

On the other hand, the stochastic expansion (11) yields the following approximation for a large $\gamma$ :

$$
T(\hat{\alpha}-\alpha) \stackrel{a}{\sim} \frac{\int_{0}^{1} \exp (c r) d W(r)}{2 \int_{0}^{1} \exp (c r) J_{c}(r) d r} .
$$

This implies that as $\gamma$ gets large the distribution of $T(\hat{\alpha}-\alpha)$ becomes less influenced by $\gamma$ and, in the limit, invariant to it. As seen from the experimental results, this is not a feature of the distribution of $T(\hat{\alpha}-\alpha)$ in finite samples. If we had worked with the stochastic expansion that excluded all terms of order $O_{p}\left(T^{-1}\right)$, i.e. considered the approximation

$$
T(\hat{\alpha}-\alpha) \stackrel{a}{\sim} X_{0}+X_{1} / T^{1 / 2}
$$

with $X_{0}$ and $X_{1}$ defined by (14) and (15), things would have been even worse. Indeed, the large $\gamma$ or large $\kappa=y_{0} / \sigma$ the approximation then becomes

$$
-\kappa\left\{\int_{0}^{1} \exp (c r) d W(r) / \int_{0}^{1} J_{c}(r)^{2} d r\right\}
$$

whose variance increases with an increase in the initial condition. This feature is opposite that present in the finite sample distribution of $T(\hat{\alpha}-\alpha)$.

\section{Conclusion}

To summarize our findings, the quality of the various approximations depends on the values of $c$ and $\gamma$. When the initial condition is zero, the continuous-time approximation which corresponds to the standard asymptotic distribution is excellent for $c>0$ as soon as $T=25$; even with as low a sample size as $T=10$ when $c$ increases. In this case, the additional corrections provided by the Edgeworth and stochastic expansions are negligeable. However, when $c \leq 0$, the Edgeworth expansion provides a significant improvement over the standard asymptotic distribution, especially when $T=25$. When the initial condition is non-zero, the continuous-time approximation is clearly the better approximation and more so as $\gamma$ increases. 


\section{Mathematical Appendix}

Proof of Theorem 1: To avoid on overburden on notation, we often omit to include each time terms like $+O\left(T^{-a}\right)$. Instead, we simply state the relation as an equality with the implicit understanding that this equality holds up to a term that vanishes at some rate and include only terms of orders that are relevant for the derivations.

We start with the expansion of the term $D(T)$ given by equation (8). Since $r$ and $s$ are the solutions to the equation $x^{2}-p x+q^{2}=0$, we have:

$$
\begin{aligned}
r, s= & \left(1+\alpha^{2}+2 \alpha \frac{u}{T}-2 \frac{v}{T^{2}}\right) / 2 \\
& \pm\left(\left(1-\alpha^{2}\right)^{2}-4 \alpha\left(1-\alpha^{2}\right) \frac{u}{T}-4\left(1-\alpha^{2}\right) \frac{u^{2}}{T^{2}}-4\left(1+\alpha^{2}\right) \frac{v}{T^{2}}-8 \alpha \frac{u v}{T^{3}}+4 \frac{v^{2}}{T^{4}}\right)^{1 / 2} / 2
\end{aligned}
$$

Since $\alpha=e^{c / T}$,

$$
\alpha=1+c / T+c^{2} /\left(2 T^{2}\right)
$$

and

$$
\alpha^{2}=1+2 c / T+2 c^{2} / T^{2} .
$$

Substituting these expansions, we have:

$$
\begin{aligned}
r= & 1+\frac{c+u}{T}+\frac{c^{2}+c u-v}{T^{2}} \\
& +\frac{i \sqrt{2}}{T}\left[\left(v-u c-c^{2} / 2\right)+\left(u v+v c-u^{2} c-2 u c^{2}-c^{3}\right) / T\right]^{1 / 2}+O\left(T^{-2}\right),
\end{aligned}
$$

and $s$ the complex conjugate of $r$. Using the notation:

$$
\begin{aligned}
& r_{1}=c+u, \\
& r_{2}=c(c+u)-v=c r_{1}-v, \\
& \delta^{2}=2 v-2 c u-c^{2}=c^{2}-2 r_{2},
\end{aligned}
$$

and the definition

$$
r=a+i b=|r| \cos \theta+i|r| \sin \theta
$$

we obtain:

$$
|r| \cos \theta=1+\frac{r_{1}}{T}+\frac{r_{2}}{T^{2}}+O\left(T^{-3}\right)
$$

and

$$
\begin{aligned}
|r| \sin \theta & =\frac{\sqrt{2}}{T}\left[\delta^{2} / 2+\left(u v+v c-u^{2} c-2 u c^{2}-c^{3}\right) / T\right]^{1 / 2}+O\left(T^{-2}\right) \\
& =\frac{\delta}{T}\left[1-\frac{2 r_{1} r_{2}}{\delta^{2} T}\right]^{1 / 2}+O\left(T^{-2}\right) .
\end{aligned}
$$


Using de Moivre's formula, we deduce that :

$$
r^{T}=|r|^{T} \cos (T \theta)+i|r|^{T} \sin (T \theta) .
$$

Note, from (8), that we can write $D(T)$ as:

$$
D(T)=2 \operatorname{Re}\left(\frac{1-\bar{r}}{r-\bar{r}} r^{T}\right)
$$

where $\bar{r}$ is the complex conjugate of $r$. After some developments we can write

$$
D(T)=|r|^{T}(\cos (T \theta)+X \sin (T \theta)),
$$

where

$$
X=(1-|r| \cos \theta) /(|r| \sin \theta)
$$

We now consider the expansion of $X$ and $\theta$. We have:

$$
\begin{aligned}
X & =-\frac{T}{\delta}\left(\frac{r_{1}}{T}+\frac{r_{2}}{T^{2}}\right)\left(1-\frac{2 r_{1} r_{2}}{\delta^{2} T}\right)^{-1 / 2} \\
& =-\frac{r_{1}}{\delta}\left(1+\frac{r_{2}}{r_{1} T}\right)\left(1+\frac{r_{1} r_{2}}{\delta^{2} T}\right) \\
& =-\frac{1}{\delta}\left[r_{1}+\frac{r_{2}}{\delta^{2} T}\left(r_{1}^{2}+\delta^{2}\right)\right]+O\left(T^{-2}\right),
\end{aligned}
$$

and

$$
\begin{aligned}
\theta & =\tan ^{-1}\left[\left(\frac{\delta}{T}\left(1-\frac{2 r_{1} r_{2}}{\delta^{2} T}\right)^{1 / 2}\right) /\left(1+\frac{r_{1}}{T}+\frac{r_{2}}{T^{2}}\right)\right] \\
& =\frac{\delta}{T}\left(1-\frac{r_{1} r_{2}}{\delta^{2} T}\right)\left(1-\frac{r_{1}}{T}-\frac{r_{2}}{T^{2}}\right) \\
& =\frac{\delta}{T}\left(1-\frac{r_{1}}{\delta^{2} T}\left(\delta^{2}+r_{2}\right)\right)+O\left(T^{-3}\right) .
\end{aligned}
$$

Hence,

$$
\cos (T \theta)=\cos \left(\delta-\frac{r_{1}}{\delta T}\left(\delta^{2}+r_{2}\right)\right)=\cos \delta+\frac{r_{1}}{\delta T}\left(\delta^{2}+r_{2}\right) \sin \delta
$$

and

$$
\sin (T \theta)=\sin \left(\delta-\frac{r_{1}}{\delta T}\left(\delta^{2}+r_{2}\right)\right)=\sin \delta-\frac{r_{1}}{\delta T}\left(\delta^{2}+r_{2}\right) \cos \delta .
$$

Since $|r|=\left((|r| \cos \theta)^{2}+(|r| \sin \theta)^{2}\right)^{1 / 2}$, we also have:

$$
\begin{aligned}
|r| & =\left[\left(1+\frac{r_{1}}{T}+\frac{r_{2}}{T^{2}}\right)^{2}+\frac{\delta^{2}}{T^{2}}\left(1-\frac{2 r_{1} r_{2}}{\delta^{2} T}\right)\right]^{1 / 2} \\
& =\left[1+\frac{2 r_{1}}{T}+\frac{r_{1}^{2}+2 r_{2}+\delta^{2}}{T^{2}}\right]^{1 / 2} \\
& =1+\frac{r_{1}}{T}+\frac{2 r_{2}+\delta^{2}}{2 T^{2}}
\end{aligned}
$$


It follows that

$$
\begin{aligned}
|r|^{T} & =\exp \left[T \ln \left(1+\frac{r_{1}}{T}+\frac{2 r_{2}+\delta^{2}}{2 T^{2}}\right)\right] \\
& =\exp \left[T\left(\frac{r_{1}}{T}+\frac{2 r_{2}+\delta^{2}}{2 T^{2}}-\frac{r_{1}^{2}}{2 T^{2}}\right)\right] \\
& =\exp \left(r_{1}\right) \exp \left(\frac{2 r_{2}+\delta^{2}-r_{1}^{2}}{2 T}\right) \\
& =\exp \left(r_{1}\right)\left[1+\frac{2 r_{2}+\delta^{2}-r_{1}^{2}}{2 T}\right]
\end{aligned}
$$

It remains to consider the expression $\cos (T \theta)+X \sin (T \theta)$. Using the results above:

$$
\begin{aligned}
\cos (T \theta)+X \sin (T \theta)= & \cos \delta+\frac{r_{1}}{\delta T}\left(\delta^{2}+r_{2}\right) \sin \delta \\
& -\frac{1}{\delta}\left[r_{1}+\frac{r_{2}}{\delta^{2} T}\left(r_{1}^{2}+\delta^{2}\right)\right]\left[\sin \delta-\frac{r_{1}}{\delta T}\left(\delta^{2}+r_{2}\right) \cos \delta\right] .
\end{aligned}
$$

Fxpanding this last expression leads to:

$$
\cos (T \theta)+X \sin (T \theta)=A_{1}-\frac{A_{2}}{\delta^{2} T}+O\left(T^{-2}\right)
$$

with

$$
\begin{aligned}
& A_{1}=\cos \delta-r_{1} \frac{\sin \delta}{\delta} \\
& A_{2}=\left(r_{2}\left(r_{1}^{2}+\delta^{2}\right)-\delta^{2} r_{1}\left(\delta^{2}+r_{2}\right)\right) \frac{\sin \delta}{\delta}-r_{1}^{2}\left(\delta^{2}+r_{2}\right) \cos \delta
\end{aligned}
$$

We finally get

$$
\begin{aligned}
D(T) & =\exp \left(r_{1}\right)\left[1+\frac{2 r_{2}+\delta^{2}-r_{1}^{2}}{2 T}\right]\left[A_{1}-\frac{A_{2}}{\delta^{2} T}\right] \\
& =\exp \left(r_{1}\right)\left\{A_{1}-\frac{1}{2 T \delta^{2}}\left(2 A_{2}-\delta^{2} A_{1}\left(2 r_{2}+\delta^{2}-r_{1}^{2}\right)\right)\right\}
\end{aligned}
$$

and we deduce that:

$$
D(T)^{-1 / 2}=A_{1}^{-1 / 2} \exp \left(-r_{1} / 2\right)\left\{1+\frac{1}{4 T \delta^{2} A_{1}}\left(2 A_{2}-\delta^{2} A_{1}\left(2 r_{2}+\delta^{2}-r_{1}^{2}\right)\right)\right\} .
$$

Fxpanding this expression, we have:

$$
\begin{aligned}
D(T)^{-1 / 2}= & \exp \left(-r_{1} / 2\right)\left(\cos \delta-r_{1} \frac{\sin \delta}{\delta}\right)^{-1 / 2} \\
& \times\left\{1+\frac{\left(r_{1}^{2}+\delta^{2}\right)\left(2 r_{2}-r_{1} \delta^{2}\right)}{4 T \delta^{2}\left(\cos \delta-r_{1} \frac{\sin \delta}{\delta}\right)} \frac{\sin \delta}{\delta}-\frac{\left(r_{1}^{2}+\delta^{2}\right)\left(2 r_{2}+\delta^{2}\right)}{4 T \delta^{2}\left(\cos \delta-r_{1} \frac{\sin \delta}{\delta}\right)} \cos \delta\right\} .
\end{aligned}
$$


We now consider the component of the moment-generating function involving $y_{0}=\kappa$ which is:

$$
\Psi=\exp \left(\kappa^{2} \frac{v}{T}-\kappa^{2} \frac{u}{T} \alpha-\frac{\kappa^{2} \alpha^{2}}{2}\right) \exp \left(\left(\frac{u}{T}+\alpha\right)^{2} \frac{\kappa^{2}}{2} \frac{D(T-1)}{D(T)}\right)
$$

Substituting $\alpha$ and $\alpha^{2}$ by the approximations (A.2) and (A.3), we have:

$$
\exp \left(\kappa^{2} \frac{v}{T}-\kappa^{2} \frac{u}{T} \alpha-\frac{\kappa^{2} \alpha^{2}}{2}\right)=\exp \left(-\frac{\kappa^{2}}{2}\right)\left\{1-\frac{\kappa^{2} r_{1}}{T}\right\}
$$

and

$$
\exp \left(\left(\frac{u}{T}+\alpha\right)^{2} \frac{\kappa^{2}}{2} \frac{D(T-1)}{D(T)}\right)=\exp \left(\frac{\kappa^{2}}{2}\right)\left\{1+\frac{\kappa^{2}\left(h+2 r_{1}\right)}{2 T}\right\},
$$

supposing that

$$
D(T-1) / D(T)=1+h / T,
$$

which is to be shown below. Hence, we have:

$$
\Psi=1+\frac{\kappa^{2} h}{2 T}+O\left(T^{-2}\right) .
$$

To determine $h$, we first expand $D(T-1)$ as follows:

$$
\begin{aligned}
D(T-1) & =|r|^{T-1}(\cos ((T-1) \theta)+X \sin ((T-1) \theta)) \\
& =|r|^{T-1}[\cos (T \theta)+\theta \sin (T \theta)+X(\sin (T \theta)-\theta \cos (T \theta))] \\
& =|r|^{T-1}\{(1-\theta X) \cos (T \theta)+(\theta+X) \sin (T \theta)\} .
\end{aligned}
$$

The expansions of $(1-\theta X)$ and $(\theta+X)$ to order $O\left(T^{-1}\right)$ are, respectively:

$$
1-\theta X=1+\frac{r_{1}}{T}
$$

and

$$
\theta+X=-\frac{r_{1}}{\delta}+\frac{1}{\delta^{3} T}\left(\delta^{4}-r_{2}\left(r_{1}^{2}+\delta^{2}\right)\right)
$$

Hence, we have:

$$
\begin{aligned}
D(T-1)= & |r|^{T-1}\left\{\left(\cos \delta-r_{1} \frac{\sin \delta}{\delta}\right)\right. \\
& \left.+\frac{1}{\delta^{2} T}\left(r_{1} \delta^{2}\left(r_{2}+\delta^{2}\right)-r_{2}\left(r_{1}^{2}+\delta^{2}\right)+\delta^{4}\right) \frac{\sin \delta}{\delta}+\frac{1}{\delta^{2} T} A_{3} \cos \delta\right\},
\end{aligned}
$$

with

$$
A_{3}=\left(r_{1} \delta^{2}+r_{1}^{2}\left(r_{2}+\delta^{2}\right)\right)
$$

We deduce that

$$
\frac{D(T-1)}{D(T)}=1+\frac{r_{1}^{2}+\delta^{2}}{T\left(\cos \delta-r_{1} \frac{\sin \delta}{\delta}\right)} \frac{\sin \delta}{\delta}=1+\frac{h}{T}
$$


with

$$
h=\frac{r_{1}^{2}+\delta^{2}}{\left(\cos \delta-r_{1} \frac{\sin \delta}{\delta}\right)} \frac{\sin \delta}{\delta} .
$$

Finally,

$$
\operatorname{MGF}(u, v)=\left(1+\frac{\kappa^{2}\left(r_{1}^{2}+\delta^{2}\right)}{2 T\left(\cos \delta-r_{1} \frac{\sin \delta}{\delta}\right)} \frac{\sin \delta}{\delta}\right) D(T)^{-1 / 2} .
$$

Combining this result with (A.4) and rearranging yields the result stated in the Theorem.

Derivation of (12). From Perron (1996), the joint moment generating function of $(Z(c, \gamma), K(c, \gamma))$ can be expressed as:

$$
M G F_{P C B}(u, v)=\exp \left(\frac{u^{2}}{4 T}-\frac{v \kappa^{2}}{T} \frac{e^{2 c}-1}{2 c}\right) M_{c, \gamma}(u, v),
$$

where

$$
M_{c, \gamma}(u, v)=\Psi_{c}(u, v) \exp \left\{-\frac{\kappa^{2} v}{2 T}(u+c-\lambda)\left[1-\exp (u+c-\lambda) \Psi_{c}^{2}(u, v)\right]\right\},
$$

with

$$
\Psi_{c}(u, v)=\{2 \lambda \exp (-(c+u)) /[(\lambda+u+c) \exp (-\lambda)+(\lambda-u-c) \exp (\lambda)]\}^{1 / 2}
$$

and

$$
\lambda^{2}=-\left(2 v-2 c u-c^{2}\right) .
$$

To obtain the alternative representation, we start with the following identities: $\exp (-\lambda)=$ $\operatorname{ch}(\lambda)-\operatorname{sh}(\lambda)$ and $\exp (\lambda)=\operatorname{ch}(\lambda)+\operatorname{sh}(\lambda)$ which define the hyperbolic functions $\operatorname{ch}(\lambda)$ et $\operatorname{sh}(\lambda)$. We can write:

$$
(\lambda+u+c) \exp (-\lambda)+(\lambda-u-c) \exp (\lambda)=2 \lambda \operatorname{ch}(\lambda)-2 r_{1} \operatorname{sh}(\lambda)
$$

with $r_{1}=c+u$ and we deduce that:

$$
\frac{(\lambda+u+c) \exp (-\lambda)+(\lambda-u-c) \exp (\lambda)}{2 \lambda}=\operatorname{ch}(\lambda)-r_{1} \frac{\operatorname{sh}(\lambda)}{\lambda} .
$$

Using $\delta=i \lambda$, we have $\delta^{2}=-\lambda^{2}=2 v-2 c u-c^{2}$ and

$$
\operatorname{ch}(\lambda)-r_{1} \frac{\operatorname{sh}(\lambda)}{\lambda}=\cos \delta-r_{1} \frac{\sin \delta}{\delta} .
$$

It is then easy to show that $\Psi_{c}(u, v)$ can be written as:

$$
\Psi_{c}(u, v)=\exp (-(c+u) / 2)\left(\cos \delta-\frac{r_{1}}{\delta} \sin \delta\right)^{-1 / 2},
$$


and we deduce that

$$
\begin{aligned}
M_{c, \gamma}(u, v)= & \exp \left(-r_{1} / 2\right)\left(\cos \delta-\frac{r_{1}}{\delta} \sin \delta\right)^{-1 / 2} \\
& \times \exp \left\{-\frac{\kappa^{2} v}{2 T}\left(r_{1}-\lambda\right)\left[1-\frac{\exp (-\lambda)}{\cos \delta-\frac{r_{1}}{\delta} \sin \delta}\right]\right\} .
\end{aligned}
$$

The expression (12) is obtained combining this last result with (A.5). 


\section{References}

[1] Bergstrom, A.R. (1984): "Continuous Time Stohastic Models and Issues of Aggregation over Time," in Z. Grilliches and M.D. Intriligator (eds.), Handbook of Econometrics, vol. 2, Nort-Holland, 1146-1210.

[2] Bobkoski, M. J. (1983): "Hypothesis Testing in Nonstationary Time Series," Unpublished Ph. D. Dissertation, Department of Statistics, University of Wisconsin.

[3] Cavanagh, C. (1986): "Roots Local to Unity," manuscript, Harvard University.

[4] Chan, N. H. and C. Z. Wei (1987): "Asymptotic Inference for Nearly Nonstationary AR(1) Processes," The Annals of Statistics 15, 1050-1063.

[5] Dickey, D. A. and Fuller, W. A. (1979): "Distribution of the Estimators for Autoregressive Time Series with a Unit Root," Journal of the American Statistical Association 74, $427-431$.

[6] Evans, G.B. and N.E. Savin (1981a): "The Calculation of the Least Squares Estimator of the Parameter in a Random Walk Model," The Annals of Statistics 9, 1114-1118.

[7] Evans, G.B. and N.E. Savin (1981b): "Testing for Unit Roots: 1," Econometrica 49, 753-778.

[8] Fuller, W.A. (1976): Introduction to Statistical Time Series, New York: John Wiley.

[9] Gurland, J. (1948): "Inversion Formulae for the Distribution of Ratios," Annals of Mathematical Statistics 19, 228-237.

[10] Hisamatsu H. and K. Maekawa (1994): "The Distribution of the Durbin - Watson Statistic in Integrated and Near-Integrated Models," Journal of Econometrics 61, 367382.

[11] Knight, J.L. and S.E. Satchell (1990): "Edgeworth Expansion of the Least Squares Estimator in the Near Integrated Model," Unpublished draft notes.

[12] Knight, J.L. and S.E. Satchell (1993): “Asymptotic Fxpansions for Random Walk with Normal Errors," Econometric Theory 9, 363-376.

[13] Mann, H.B. and A. Wald (1943): "On the Statistical Treatment of Linear Stochastic Difference Equations," Econometrica 11, 173-220.

[14] Perron, P. (1989): "The Calculation of the Limiting Distribution of the Least Squares Estimator in a Near-Integrated Model," Econometric Theory 5, 241-255. 
[15] Perron, P. (1991a): "A Continuous Time Approximation to the Unstable First-order Autoregressive Process: The Case Without an Intercept," Econometrica 59, 211-236.

[16] Perron, P. (1991b): "A Continuous Time Approximation to the Stationary First-order Autoregressive Model," Econometric Theory 7, 236-252.

[17] Perron, P. (1996): "The Adequacy of Asymptotic Approximations in The NearIntegrated Autoregressive Model with Dependent Errors," Journal of Econometrics 70, $317-350$.

[18] Phillips, P.C.B. (1977): “Approximations to Some Finite Sample Distributions Associated with a First Order Stochastic Difference Equation," Econometrica 45, 463-485.

[19] Phillips, P.C.B. (1987a): “Time Series Regression wiith Unit Roots," Econometrica 55, 277-302.

[20] Phillips, P.C.B. (1987b): “Towards a Unified Asymptotic Theory for Autoregression," Biometrika 74, 535-547.

[21] Phillips, P.C.B. (1987c): “Asymptotic Expansions in Nonstationary Vector Autoregressions," Econometric Theory 3, 45-68.

[22] Phillips, P.C.B. and P. Perron (1988): “Testing for a Unit Root in Time Series Regression," Biometrika 75, 335-346.

[23] Rubin, H. (1950): "Consistency of Maximum Likelihood Estimates in The Explosive Case," in T. C. Koopmans (ed.), Statistical Inference in Dynamic Economic Models, New York: John Wiley.

[24] Satchell, S.E. (1984): "Approximation to the Finite Sample Distribution For Nonstable First Order Stochastic Difference Equations," Econometrica 52, 1271-1289.

[25] Vodounou, C. (1997a): Essais en économetrie théorique et en économétrie de la finance appliqués aux modèles dynamiques, Unpublished PhD Dissertation, Département de Sciences Économiques, Université de Montréal.

[26] Vodounou, C. (1997b): “Adéquation des approximations d'Edgeworth dans le modèle $\operatorname{AR}(1)$ sable avec valeur initiale fixe," manuscript, Université de Montréal.

[27] White, J.S.(1958): "The Limiting Distribution of the Serial Correlation Coefficient in the Explosive Case," Annals of Mathematical Statistics 29, 1188-1197. 
Table 1: Critical values of the exact distribution of $T(\hat{\alpha}-\alpha)$

\begin{tabular}{|c|c|c|c|c|c|c|c|c|}
\hline & $1 \%$ & $2.5 \%$ & $5 \%$ & $\overline{10 \%}$ & $\overline{90 \%}$ & $95 \%$ & $\overline{97.5 \%}$ & $\overline{99 \%}$ \\
\hline \multicolumn{9}{|c|}{$c=-5$} \\
\hline \multicolumn{9}{|l|}{$\gamma=0.0$} \\
\hline $\mathrm{T}=10$ & -9.125 & -7.658 & -6.381 & -4.964 & 2.330 & 2.907 & 3.475 & 4.254 \\
\hline $\mathrm{T}=25$ & -12.825 & -10.228 & -8.363 & -6.396 & 2.395 & 3.000 & 3.438 & 4.028 \\
\hline $\mathrm{T}=50$ & -14.671 & -11.681 & -9.442 & -7.042 & 2.431 & 3.001 & 3.455 & 3.972 \\
\hline $\mathrm{T}=100$ & -15.758 & -12.408 & -9.913 & -7.344 & 2.444 & 3.033 & 3.478 & 3.992 \\
\hline \multicolumn{9}{|l|}{$\gamma=0.5$} \\
\hline $\mathrm{T}=10$ & -7.750 & -6.555 & -5.442 & -4.225 & 2.065 & 2.556 & 2.962 & 3.436 \\
\hline $\mathrm{T}=25$ & -10.539 & -8.711 & -7.123 & -5.421 & 2.165 & 2.722 & 3.143 & 3.578 \\
\hline $\mathrm{T}=50$ & -12.061 & -9.530 & -7.840 & -5.935 & 2.213 & 2.772 & 3.206 & 3.646 \\
\hline $\mathrm{T}=100$ & -13.305 & -10.487 & -8.314 & -6.266 & 2.212 & 2.777 & 3.193 & 3.614 \\
\hline \multicolumn{9}{|l|}{$\gamma=1.0$} \\
\hline $\mathrm{T}=10$ & -5.642 & -4.756 & -3.941 & -3.050 & 1.693 & 2.079 & 2.395 & 2.713 \\
\hline $\mathrm{T}=25$ & -7.407 & -6.053 & -5.002 & -3.878 & 1.815 & 2.260 & 2.610 & 2.930 \\
\hline $\mathrm{T}=50$ & -8.488 & -6.930 & -5.616 & -4.228 & 1.862 & 2.335 & 2.677 & 3.038 \\
\hline $\mathrm{T}=100$ & -8.969 & -7.193 & -5.956 & -4.464 & 1.869 & 2.341 & 2.713 & 3.056 \\
\hline \multicolumn{9}{|l|}{$\gamma=2.0$} \\
\hline $\mathrm{T}=10$ & -3.282 & -2.708 & -2.245 & -1.727 & 1.167 & 1.464 & 1.694 & 1.939 \\
\hline $\mathrm{T}=25$ & -4.048 & -3.308 & -2.708 & -2.099 & 1.278 & 1.594 & 1.869 & 2.152 \\
\hline $\mathrm{T}=50$ & -4.462 & -3.683 & -3.031 & -2.317 & 1.323 & 1.656 & 1.943 & 2.232 \\
\hline $\mathrm{T}=100$ & & -3.831 & -3.117 & $\begin{array}{l}-2.385 \\
=0\end{array}$ & 1.333 & 1.675 & 1.956 & 2.251 \\
\hline$\gamma=0.0$ & & & & & & & & \\
\hline $\mathrm{T}=10$ & -9.915 & -8.065 & -6.594 & -4.907 & 1.171 & 1.633 & 2.111 & 2.777 \\
\hline $\mathrm{T}=25$ & -11.822 & -9.408 & -7.397 & -5.348 & 1.012 & 1.408 & 1.788 & 2.321 \\
\hline $\mathrm{T}=50$ & -12.577 & -9.926 & -7.653 & -5.523 & 0.972 & 1.337 & 1.678 & 2.108 \\
\hline $\begin{array}{l}\mathrm{T}=100 \\
\gamma=0.5\end{array}$ & -13.364 & -10.255 & -7.928 & -5.680 & 0.959 & 1.312 & 1.652 & 2.084 \\
\hline $\mathrm{T}=10$ & -7.892 & -6.392 & -5.230 & -3.897 & 0.864 & 1.188 & 1.508 & 1.915 \\
\hline $\mathrm{T}=25$ & -9.589 & -7.578 & -5.897 & -4.347 & 0.791 & 1.098 & 1.372 & 1.765 \\
\hline $\mathrm{T}=50$ & -9.954 & -7.973 & -6.128 & -4.454 & 0.797 & 1.092 & 1.348 & 1.688 \\
\hline $\begin{array}{l}\mathrm{T}=100 \\
\gamma=1.0\end{array}$ & -10.457 & -8.159 & -6.417 & -4.587 & 0.772 & 1.052 & 1.313 & 1.608 \\
\hline $\mathrm{T}=10$ & -5.253 & -4.209 & -3.364 & -2.469 & 0.634 & 0.843 & 1.001 & 1.221 \\
\hline $\mathrm{T}=25$ & -5.892 & -4.638 & -3.677 & -2.653 & 0.597 & 0.793 & 0.961 & 1.167 \\
\hline $\mathrm{T}=50$ & -6.557 & -5.128 & -3.995 & -2.788 & 0.598 & 0.798 & 0.955 & 1.156 \\
\hline $\mathrm{T}=100$ & -6.629 & -5.178 & -4.063 & -2.891 & 0.588 & 0.777 & 0.935 & 1.125 \\
\hline
\end{tabular}


Table 1 (cont'd) : Critical values of the exact distribution of $T(\widehat{\alpha}-\alpha)$

\begin{tabular}{lcccccccc}
\hline \hline & $\mathbf{1 \%}$ & $\mathbf{2 . 5 \%}$ & $\mathbf{5 \%}$ & $\mathbf{1 0 \%}$ & $\mathbf{9 0 \%}$ & $\mathbf{9 5 \%}$ & $\mathbf{9 7 . 5 \%}$ & $\mathbf{9 9 \%}$ \\
\hline$\gamma=2.0$ & & & & $\mathbf{c}=\mathbf{0}$ & & & & \\
$\mathrm{T}=10$ & -2.433 & -1.873 & -1.462 & -1.057 & 0.430 & 0.549 & 0.648 & 0.758 \\
$\mathrm{~T}=25$ & -2.589 & -1.996 & -1.569 & -1.102 & 0.417 & 0.531 & 0.630 & 0.737 \\
$\mathrm{~T}=50$ & -2.715 & -2.055 & -1.582 & -1.131 & 0.416 & 0.534 & 0.629 & 0.738 \\
$\mathrm{~T}=100$ & -2.721 & -2.107 & -1.613 & -1.151 & 0.411 & 0.527 & 0.617 & 0.718 \\
$\gamma=0.0$ & & & & $\mathbf{c = 2}$ & & & & \\
$\mathrm{T}=10$ & -10.056 & -7.941 & -6.168 & -4.280 & 0.740 & 1.103 & 1.479 & 2.107 \\
$\mathrm{~T}=25$ & -11.216 & -8.423 & -6.308 & -4.296 & 0.590 & 0.877 & 1.180 & 1.602 \\
$\mathrm{~T}=50$ & -11.766 & -8.736 & -6.527 & -4.370 & 0.553 & 0.807 & 1.078 & 1.430 \\
$\mathrm{~T}=100$ & -11.740 & -8.765 & -6.551 & -4.385 & 0.545 & 0.802 & 1.065 & 1.423 \\
$\gamma=0.5$ & & & & & & & & \\
$\mathrm{~T}=10$ & -7.598 & -5.796 & -4.461 & -2.832 & 0.459 & 0.655 & 0.867 & 1.163 \\
$\mathrm{~T}=25$ & -8.423 & -6.302 & -4.686 & -2.962 & 0.406 & 0.583 & 0.770 & 1.054 \\
$\mathrm{~T}=50$ & -8.953 & -6.493 & -4.751 & -3.016 & 0.401 & 0.565 & 0.745 & 1.008 \\
$\mathrm{~T}=100$ & -9.207 & -6.595 & -4.801 & -3.000 & 0.377 & 0.550 & 0.718 & 0.957 \\
$\gamma=1.0$ & & & & & & & & \\
$\mathrm{~T}=10$ & -4.359 & -2.564 & -1.421 & -0.788 & 0.290 & 0.386 & 0.481 & 0.611 \\
$\mathrm{~T}=25$ & -4.306 & -2.870 & -1.617 & -0.833 & 0.263 & 0.356 & 0.445 & 0.558 \\
$\mathrm{~T}=50$ & -4.703 & -3.036 & -1.676 & -0.860 & 0.262 & 0.348 & 0.432 & 0.543 \\
$\mathrm{~T}=100$ & -5.037 & -3.163 & -1.735 & -0.887 & 0.252 & 0.340 & 0.418 & 0.526 \\
$\gamma=2.0$ & & & & & & & & \\
$\mathrm{~T}=10$ & -0.562 & -0.437 & -0.338 & -0.247 & 0.165 & 0.214 & 0.257 & 0.311 \\
$\mathrm{~T}=25$ & -0.585 & -0.432 & -0.334 & -0.241 & 0.154 & 0.200 & 0.237 & 0.288 \\
$\mathrm{~T}=50$ & -0.596 & -0.445 & -0.335 & -0.242 & 0.151 & 0.196 & 0.235 & 0.279 \\
$\mathrm{~T}=100$ & -0.597 & -0.424 & -0.325 & -0.238 & 0.149 & 0.192 & 0.226 & 0.272 \\
& & & & & & & & \\
\hline
\end{tabular}


Table 2: Critical values from the Edgeworth approximation

\begin{tabular}{lllllllll}
\hline \hline & $\mathbf{1 \%}$ & $\mathbf{2 . 5 \%}$ & $\mathbf{5 \%}$ & $\mathbf{1 0 \%}$ & $\mathbf{9 0 \%}$ & $\mathbf{9 5 \%}$ & $\mathbf{9 7 . 5 \%}$ & $\mathbf{9 9 \%}$ \\
\hline & & \multicolumn{7}{c}{$\mathbf{c}=\mathbf{- 5}$} \\
$\gamma=0.0$ & & & & & & & \\
$\mathrm{~T}=10$ & -7.343 & -6.709 & -5.903 & -4.751 & 2.276 & 2.820 & 3.260 & 3.905 \\
$\mathrm{~T}=25$ & -11.838 & -9.980 & -8.263 & -6.310 & 2.380 & 2.968 & 3.432 & 4.001 \\
$\mathrm{~T}=50$ & -14.244 & -11.526 & -9.316 & -6.980 & 2.418 & 3.017 & 3.480 & 4.019 \\
$\mathrm{~T}=100$ & -15.515 & -12.349 & -9.881 & -7.344 & 2.437 & 3.040 & 3.503 & 4.027 \\
$\gamma=0.5$ & & & & & & & & \\
$\mathrm{~T}=10$ & -6.418 & -5.930 & -5.278 & -4.302 & 2.103 & 2.562 & 2.886 & 3.190 \\
$\mathrm{~T}=25$ & -9.066 & -7.993 & -6.820 & -5.336 & 2.187 & 2.692 & 3.057 & 3.414 \\
$\mathrm{~T}=50$ & -10.553 & -9.010 & -7.518 & -5.775 & 2.218 & 2.738 & 3.113 & 3.481 \\
$\mathrm{~T}=100$ & -11.478 & -9.600 & -7.909 & -6.016 & 2.233 & 2.761 & 3.141 & 3.513 \\
$\gamma=1.0$ & & & & & & & & \\
$\mathrm{~T}=10$ & -3.354 & -3.194 & -2.948 & -2.511 & 1.735 & 2.038 & 2.214 & 2.333 \\
$\mathrm{~T}=25$ & -4.182 & -3.952 & -3.612 & -3.037 & 1.775 & 2.104 & 2.299 & 2.433 \\
$\mathrm{~T}=50$ & -4.557 & -4.289 & -3.897 & -3.255 & 1.790 & 2.129 & 2.330 & 2.469 \\
$\mathrm{~T}=100$ & -4.819 & -4.520 & -4.091 & -3.401 & 1.798 & 2.141 & 2.346 & 2.488 \\
$\gamma=2.0$ & & & & & & & & \\
$\mathrm{~T}=10$ & -1.099 & -1.055 & -0.982 & -0.839 & 1.123 & 1.249 & 1.314 & 1.354 \\
$\mathrm{~T}=25$ & -1.188 & -1.140 & -1.063 & -0.911 & 1.149 & 1.282 & 1.351 & 1.393 \\
$\mathrm{~T}=50$ & -1.220 & -1.171 & -1.091 & -0.937 & 1.158 & 1.294 & 1.364 & 1.407 \\
$\mathrm{~T}=100$ & -1.236 & -1.187 & -1.106 & -0.950 & 1.163 & 1.300 & 1.371 & 1.415 \\
\hline
\end{tabular}


Table 2 (cont'd): Critical values from the Edgeworth approximation

\begin{tabular}{lllllllll}
\hline \hline & $\mathbf{1 \%}$ & $\mathbf{2 . 5 \%}$ & $\mathbf{5 \%}$ & $\mathbf{1 0 \%}$ & $\mathbf{9 0 \%}$ & $\mathbf{9 5 \%}$ & $\mathbf{9 7 . 5 \%}$ & $\mathbf{9 9 \%}$ \\
\hline$\gamma=0.0$ & & & $\mathbf{c}=\mathbf{0 . 0}$ & & & & \\
$\mathrm{T}=10$ & -9.420 & -7.920 & -6.500 & -4.870 & 1.140 & 1.570 & 1.980 & 2.480 \\
$\mathrm{~T}=25$ & -11.770 & -9.320 & -7.360 & -5.350 & 1.010 & 1.400 & 1.770 & 2.250 \\
$\mathrm{~T}=50$ & -12.710 & -9.860 & -7.690 & -5.520 & 0.970 & 1.340 & 1.690 & 2.150 \\
$\mathrm{~T}=100$ & -13.210 & -10.140 & -7.860 & -5.610 & 0.950 & 1.310 & 1.650 & 2.090 \\
$\gamma=0.5$ & & & & & & & & \\
$\mathrm{~T}=10$ & -6.597 & -5.802 & -4.904 & -3.768 & 0.892 & 1.249 & 1.611 & 2.124 \\
$\mathrm{~T}=25$ & -8.035 & -6.742 & -5.501 & -4.098 & 0.789 & 1.077 & 1.337 & 1.678 \\
$\mathrm{~T}=50$ & -8.728 & -7.145 & -5.740 & -4.223 & 0.758 & 1.027 & 1.257 & 1.532 \\
$\mathrm{~T}=100$ & -9.130 & -7.367 & -5.868 & -4.288 & 0.743 & 1.003 & 1.219 & 1.467 \\
& & & & $\mathbf{c}=\mathbf{2}$ & & & & \\
$\gamma=0.0$ & & & & & & & & \\
$\mathrm{~T}=10$ & -9.034 & -7.457 & -5.815 & -4.046 & 0.702 & 1.014 & 1.342 & 1.800 \\
$\mathrm{~T}=25$ & -10.828 & -8.247 & -6.233 & -4.216 & 0.591 & 0.865 & 1.152 & 1.561 \\
$\mathrm{~T}=50$ & -11.422 & -8.552 & -6.392 & -4.278 & 0.555 & 0.812 & 1.081 & 1.463 \\
$\mathrm{~T}=100$ & -11.734 & -8.711 & -6.474 & -4.311 & 0.537 & 0.785 & 1.044 & 1.410 \\
$\gamma=0.5$ & & & & & & & & \\
$\mathrm{~T}=10$ & -6.034 & -5.001 & -3.924 & -2.617 & 0.473 & 0.712 & 0.992 & 1.432 \\
$\mathrm{~T}=25$ & -6.883 & -5.460 & -4.151 & -2.678 & 0.387 & 0.549 & 0.718 & 0.969 \\
$\mathrm{~T}=50$ & -7.266 & -5.646 & -4.238 & -2.701 & 0.364 & 0.506 & 0.642 & 0.820 \\
$\mathrm{~T}=100$ & -7.484 & -5.748 & -4.283 & -2.713 & 0.353 & 0.486 & 0.609 & 0.757 \\
$\gamma=1.0$ & & & & & & & & \\
$\mathrm{~T}=10$ & -0.625 & -0.582 & -0.520 & -0.424 & 0.205 & 0.244 & 0.267 & 0.281 \\
$\mathrm{~T}=25$ & -0.617 & -0.575 & -0.515 & -0.421 & 0.187 & 0.223 & 0.242 & 0.255 \\
$\mathrm{~T}=50$ & -0.606 & -0.565 & -0.507 & -0.416 & 0.182 & 0.216 & 0.235 & 0.247 \\
$\mathrm{~T}=100$ & -0.601 & -0.561 & -0.504 & -0.413 & 0.179 & 0.213 & 0.232 & 0.243 \\
& & & & & & & & \\
\hline & & & & & & & &
\end{tabular}


Table 3: Critical values from the continuous-time approximation

\begin{tabular}{|c|c|c|c|c|c|c|c|c|}
\hline & $1 \%$ & $2.5 \%$ & $5 \%$ & $10 \%$ & $90 \%$ & $95 \%$ & $97.5 \%$ & $99 \%$ \\
\hline \multicolumn{9}{|c|}{$c=-5$} \\
\hline$\gamma=0.0$ & -16.738 & -13.174 & -10.463 & -7.724 & 2.456 & 3.064 & 3.524 & 4.034 \\
\hline 0.5 & -13.896 & -10.983 & -8.7550 & -6.490 & 2.270 & 2.826 & 3.241 & 3.679 \\
\hline 1.0 & -9.541 & -7.610 & -6.1140 & -4.570 & 1.928 & 2.402 & 2.758 & 3.122 \\
\hline 2.0 & -4.874 & -3.953 & -3.221 & -2.441 & 1.384 & 1.736 & 2.012 & 2.304 \\
\hline \multicolumn{9}{|c|}{$c=0.0$} \\
\hline$\gamma=0.0$ & -13.692 & -10.440 & -8.038 & -5.713 & 0.928 & 1.285 & 1.612 & 2.033 \\
\hline 0.5 & -10.957 & -8.354 & -6.432 & -4.571 & 0.764 & 1.048 & 1.304 & 1.634 \\
\hline 1.0 & -6.848 & -5.220 & -4.020 & -2.857 & 0.588 & 0.778 & 0.938 & 1.130 \\
\hline 2.0 & -2.739 & -2.089 & -1.609 & -1.145 & 0.412 & 0.527 & 0.621 & 0.726 \\
\hline \multicolumn{9}{|c|}{$\mathrm{c}=2$} \\
\hline$\gamma=0.0$ & -12.056 & -8.876 & -6.559 & -4.343 & 0.519 & 0.759 & 1.007 & 1.355 \\
\hline 0.5 & -9.255 & -6.708 & -4.844 & -3.021 & 0.376 & 0.540 & 0.714 & 0.968 \\
\hline 1.0 & -4.876 & -3.126 & -1.740 & -0.878 & 0.249 & 0.336 & 0.418 & 0.529 \\
\hline 2.0 & -0.590 & -0.431 & -0.329 & -0.237 & 0.147 & 0.190 & 0.227 & 0.271 \\
\hline
\end{tabular}

Table 4: Critical values from the stochastic expansion

\begin{tabular}{lcccccccc}
\hline \hline & $\mathbf{1 \%}$ & $\mathbf{2 . 5 \%}$ & $\mathbf{5 \%}$ & $\mathbf{1 0 \%}$ & $\mathbf{9 0 \%}$ & $\mathbf{9 5 \%}$ & $\mathbf{9 7 . 5 \%}$ & $\mathbf{9 9 \%}$ \\
\hline & & \multicolumn{7}{c}{$\mathbf{c}=\mathbf{5 . 0}$} \\
$\mathrm{T}=10.0$ & -22.949 & -18.030 & -14.095 & -9.917 & 3.588 & 4.418 & 5.174 & 6.213 \\
$\mathrm{~T}=25$ & -19.776 & -15.286 & -11.873 & -8.476 & 2.898 & 3.542 & 4.045 & 4.638 \\
$\mathrm{~T}=50$ & -18.350 & -14.233 & -11.145 & -8.078 & 2.668 & 3.285 & 3.757 & 4.292 \\
$\mathrm{~T}=100$ & -17.559 & -13.701 & -10.797 & -7.896 & 2.559 & 3.170 & 3.634 & 4.155 \\
& & & $\mathbf{c}=\mathbf{0 . 0}$ & & & & \\
$\gamma=0.0$ & & & & & & & \\
$\mathrm{~T}=10$ & -15.740 & -12.140 & -8.780 & -5.800 & 1.030 & 1.410 & 1.780 & 2.270 \\
$\mathrm{~T}=25$ & -14.800 & -11.070 & -8.300 & -5.750 & 0.970 & 1.340 & 1.680 & 2.140 \\
$\mathrm{~T}=50$ & -14.270 & -10.730 & -8.160 & -5.730 & 0.950 & 1.310 & 1.650 & 2.080 \\
$\mathrm{~T}=100$ & -14.000 & -10.140 & -8.100 & -5.720 & 0.940 & 1.300 & 1.630 & 2.060 \\
& & & & $\mathbf{c}=\mathbf{2}$ & & & & \\
$\gamma=0.0$ & & & & & & & & \\
$\mathrm{~T}=10$ & -13.599 & -9.257 & -6.460 & -4.136 & 0.541 & 0.796 & 1.068 & 1.459 \\
$\mathrm{~T}=25$ & -12.612 & -9.013 & -6.525 & -4.262 & 0.528 & 0.773 & 1.031 & 1.397 \\
$\mathrm{~T}=50$ & -12.319 & -8.942 & -6.543 & -4.303 & 0.524 & 0.766 & 1.019 & 1.376 \\
$\mathrm{~T}=100$ & -12.188 & -8.909 & -6.551 & -4.323 & 0.521 & 0.762 & 1.013 & 1.366 \\
\hline
\end{tabular}



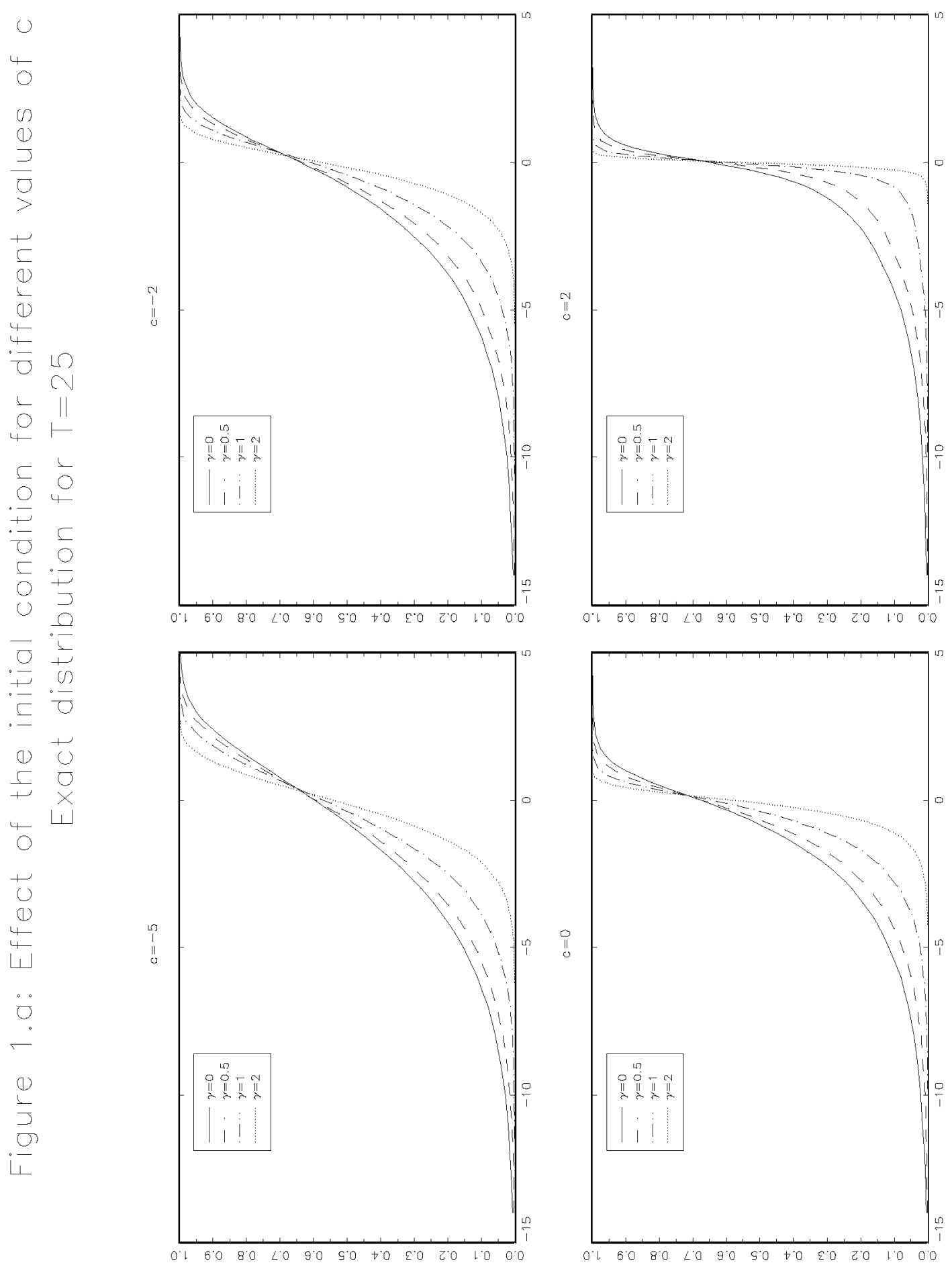

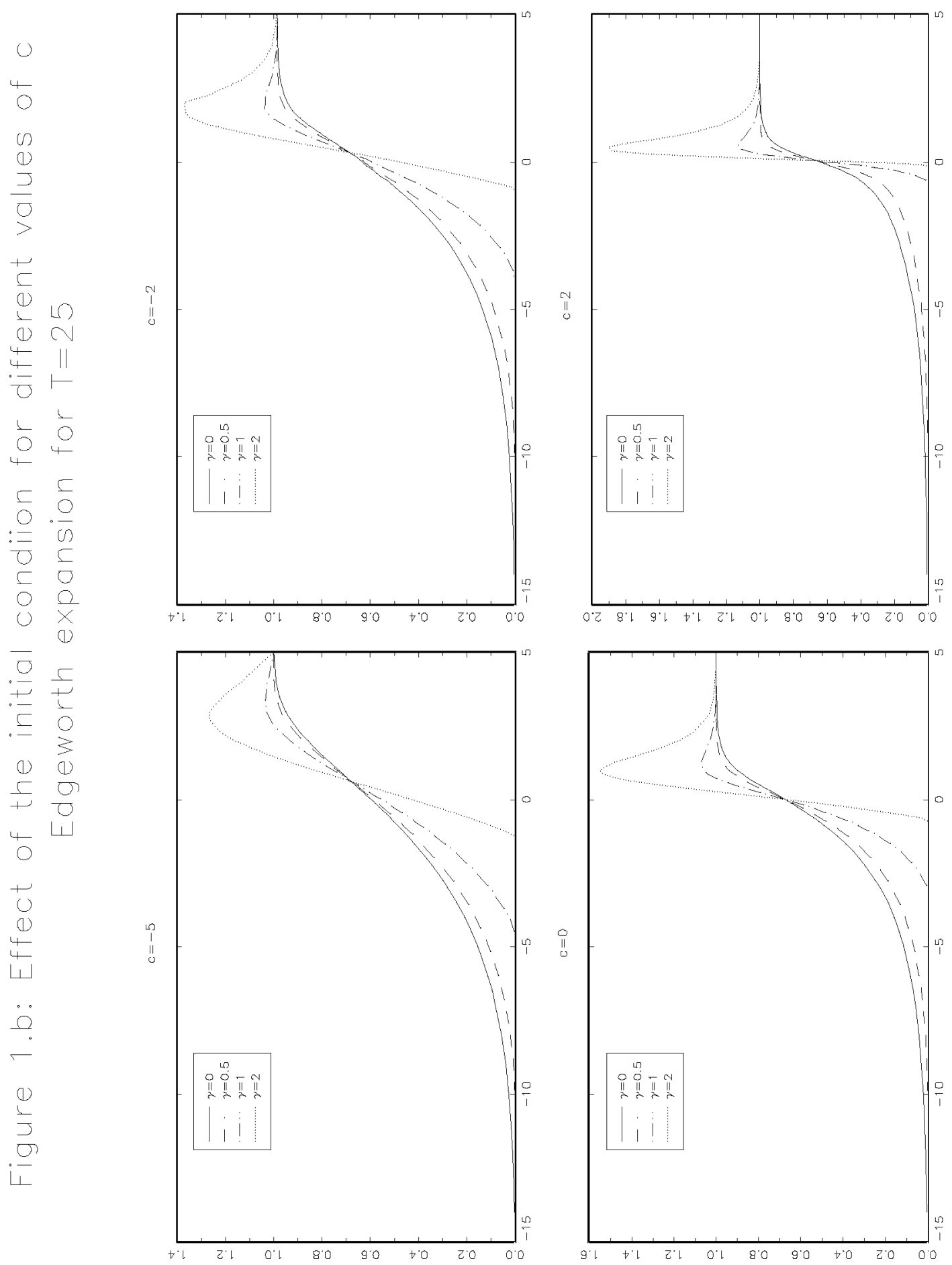


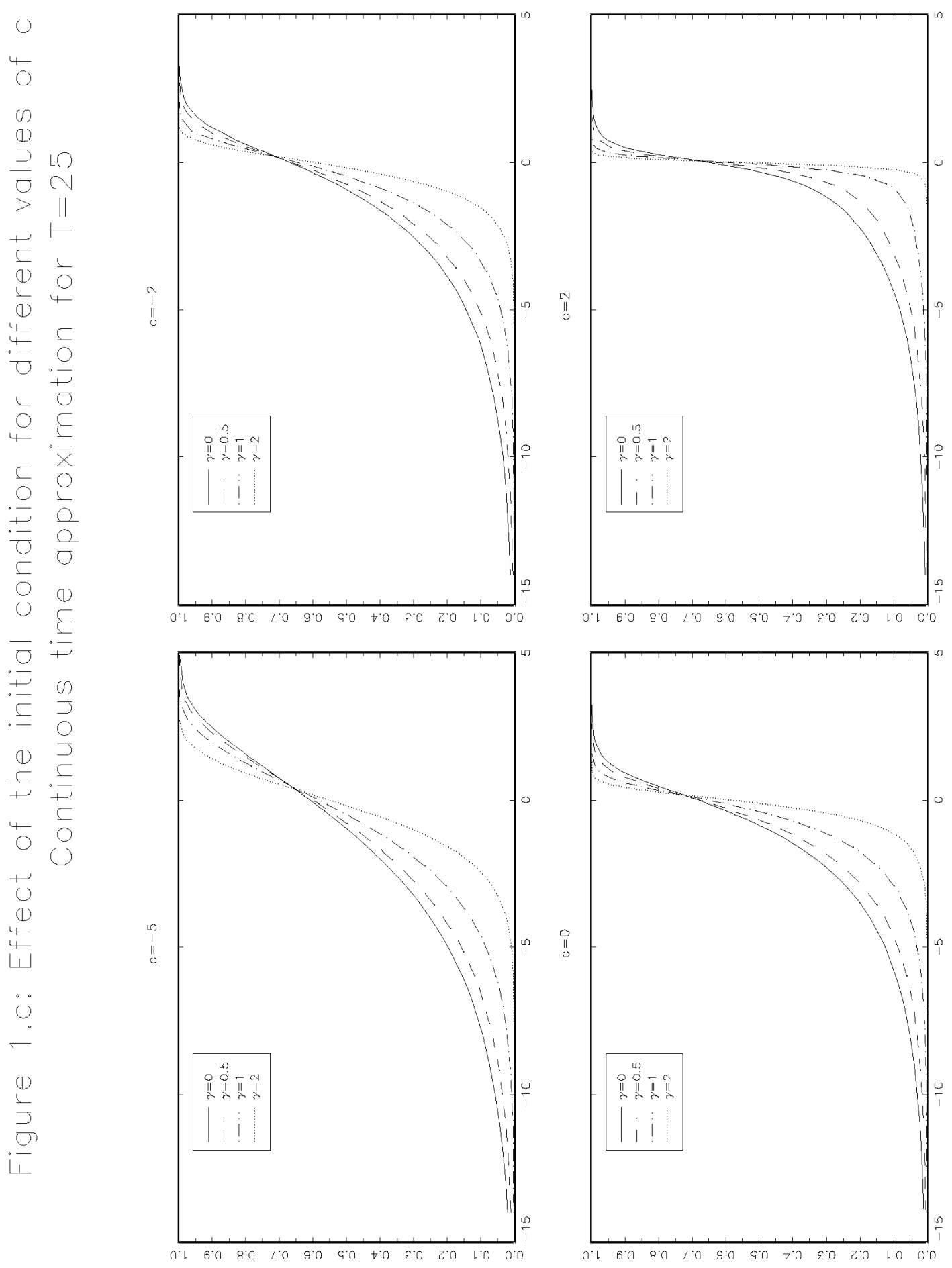




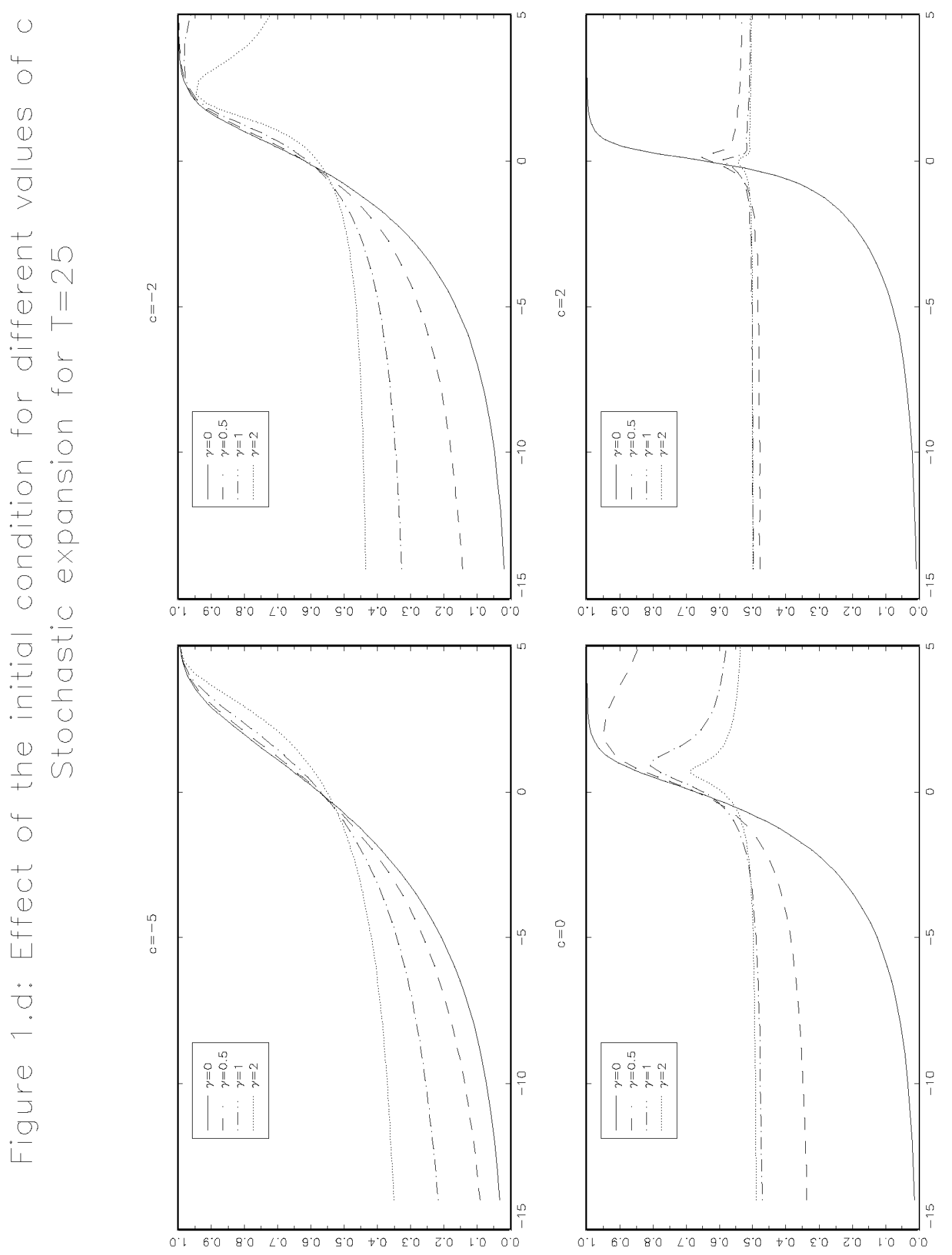



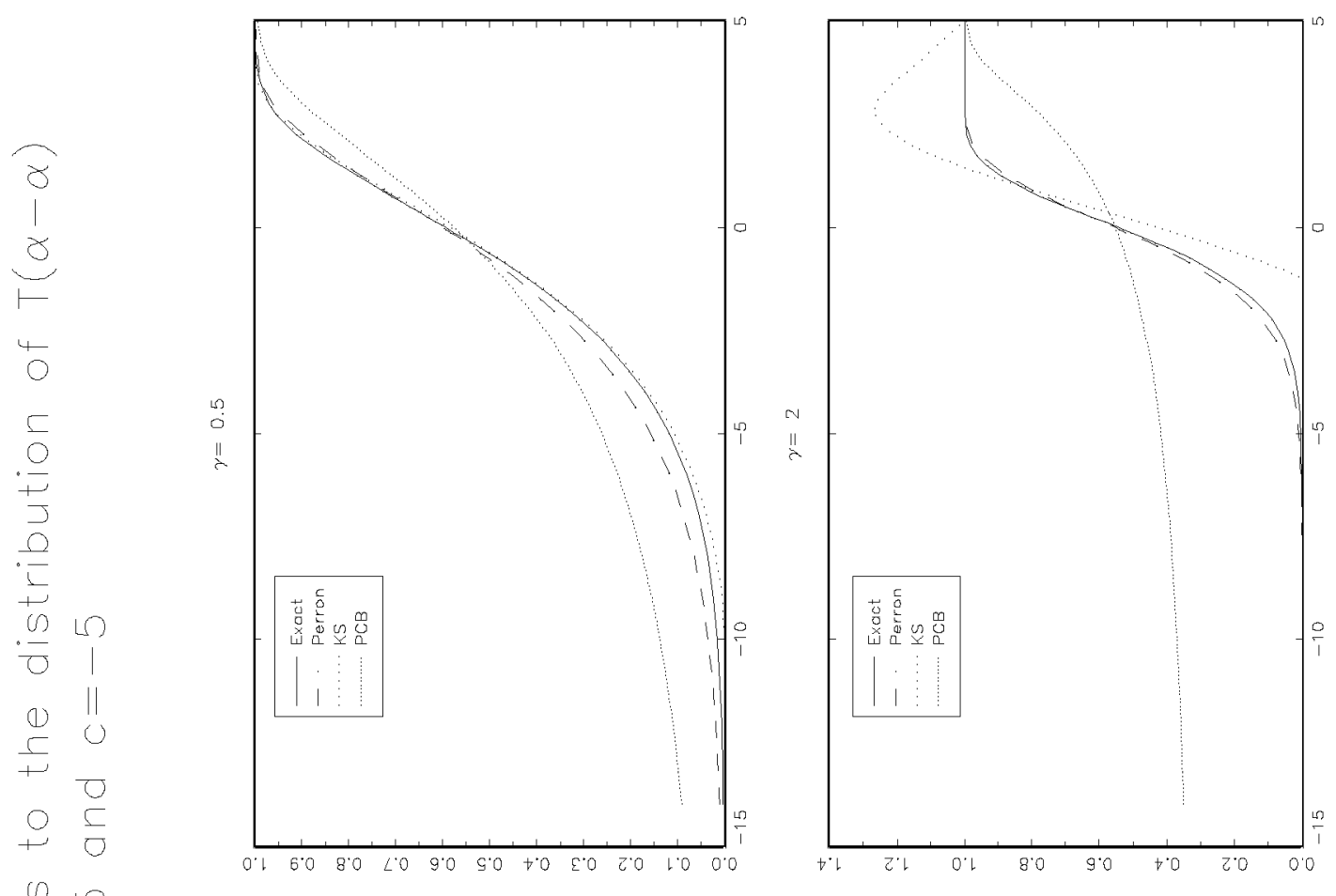

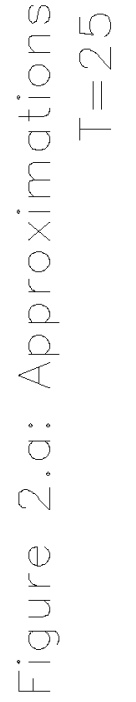
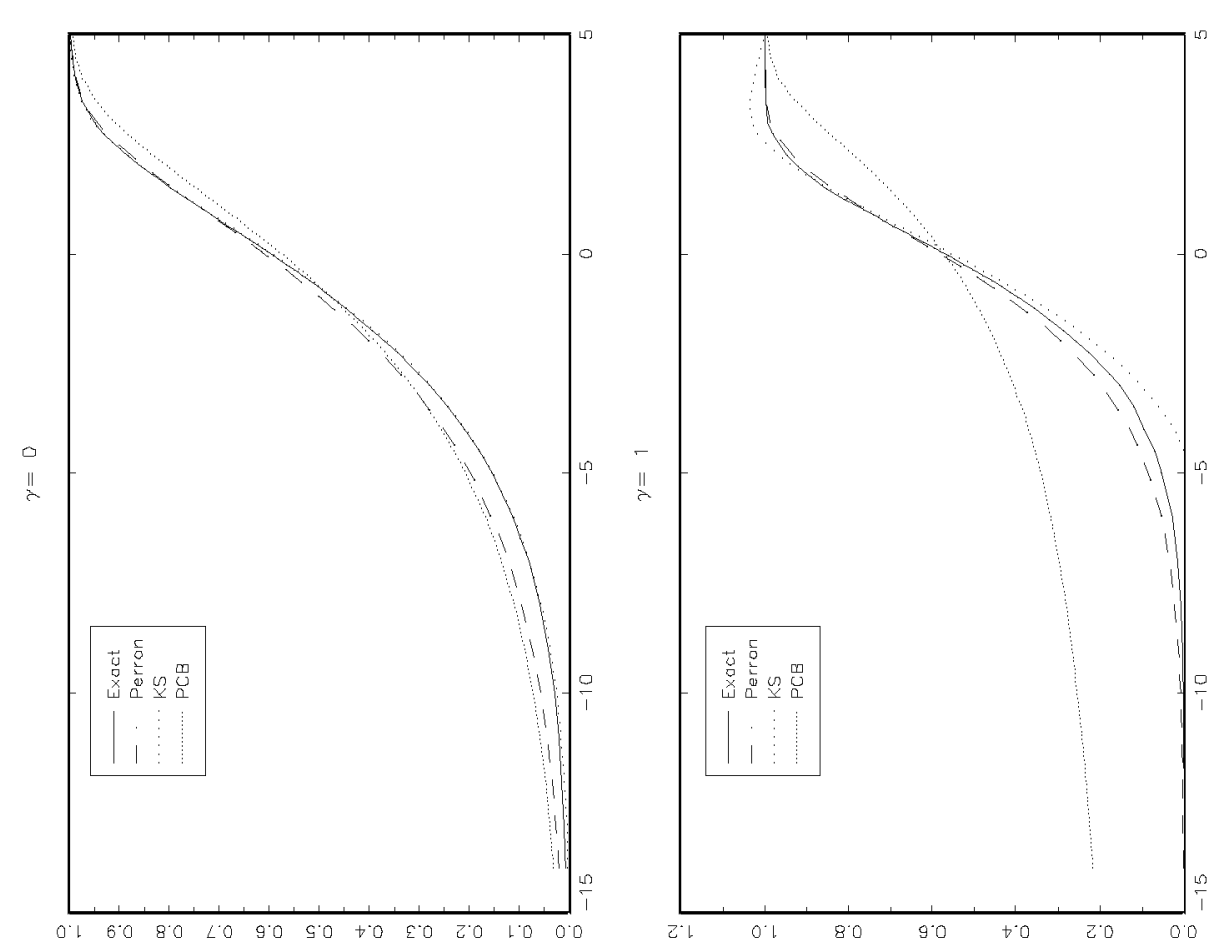

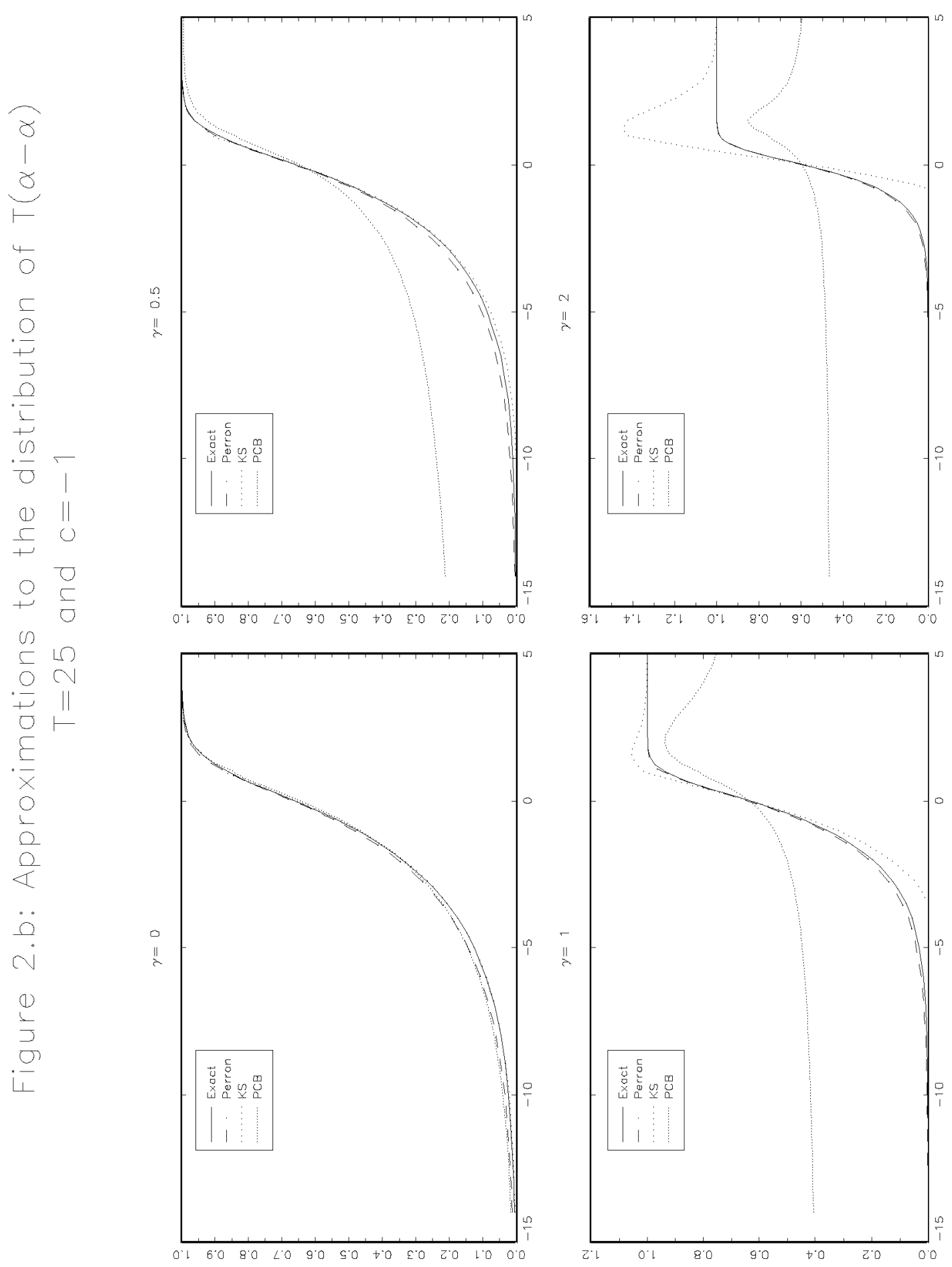

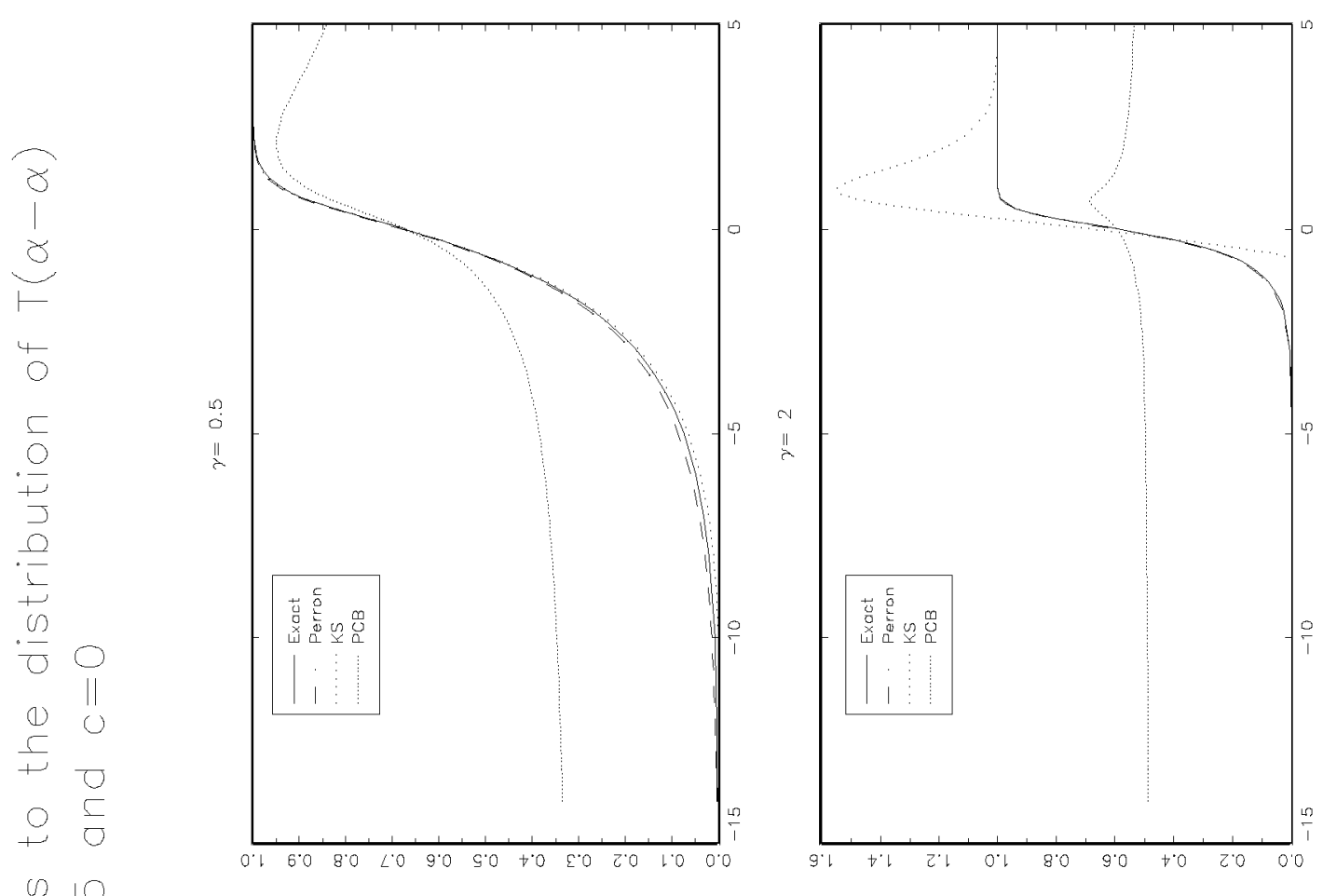

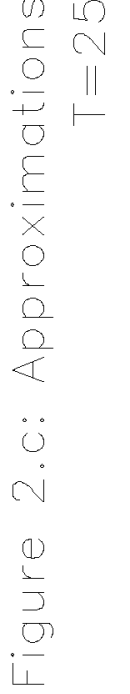
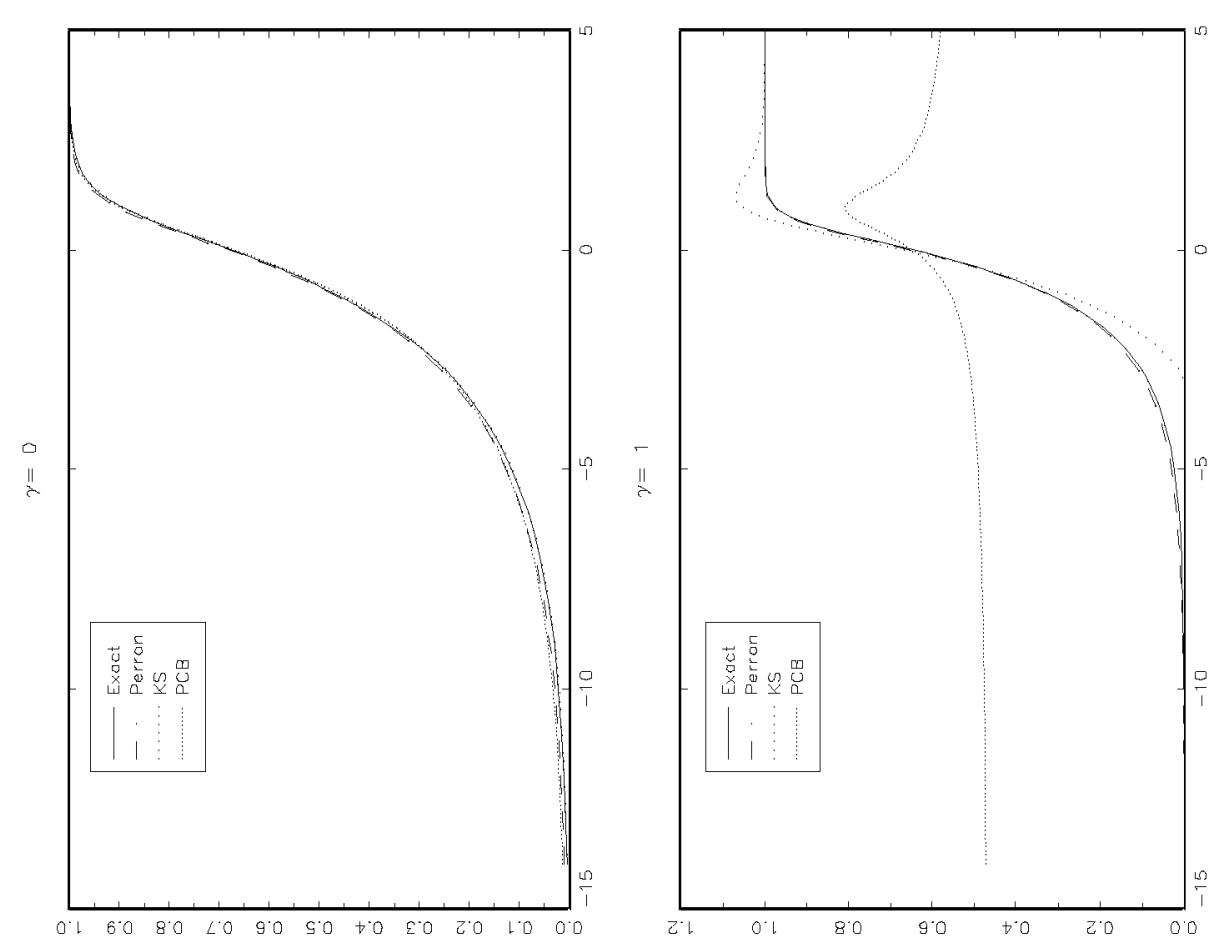

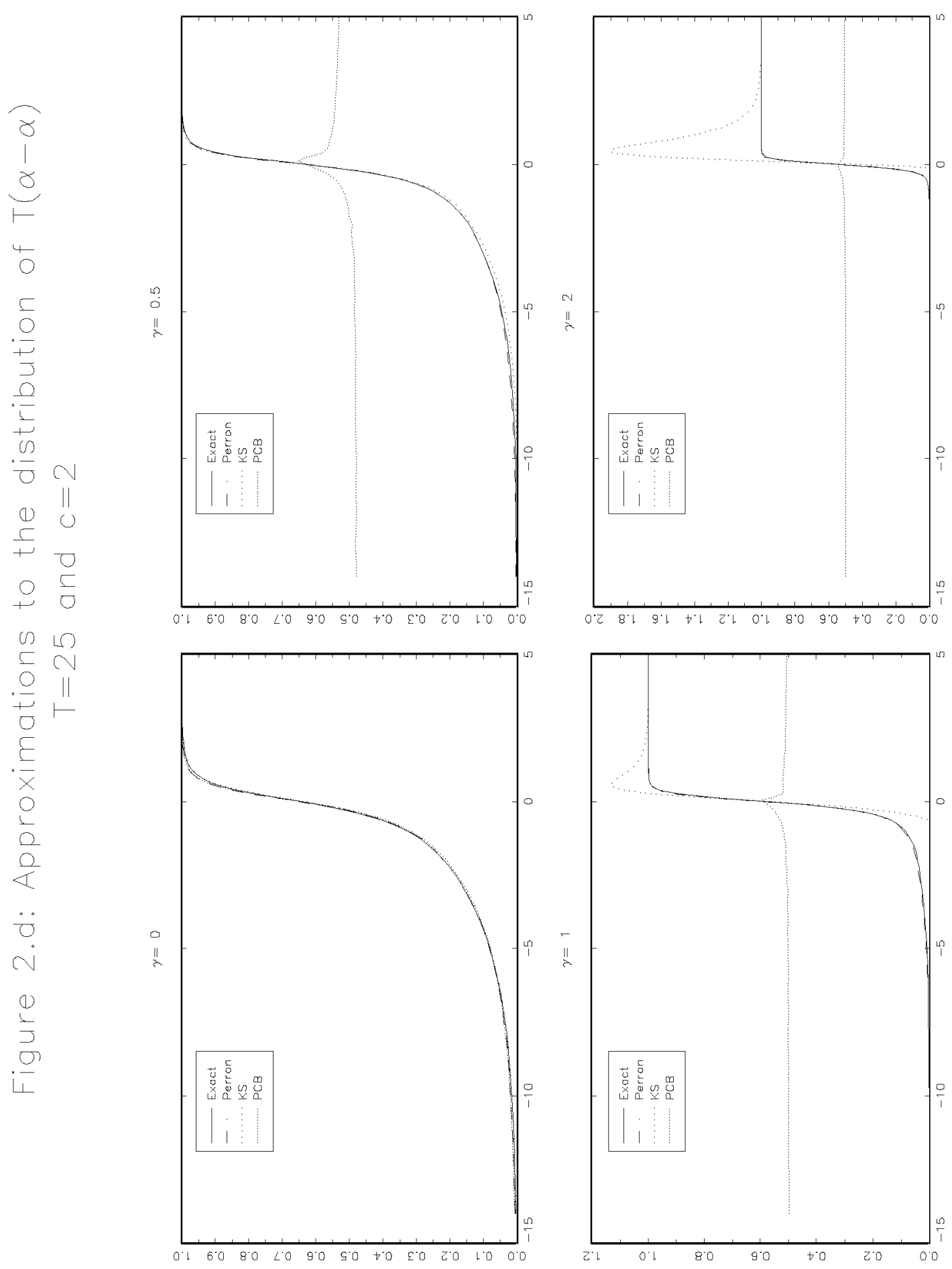